\title{
Imaging Technologies for Biomedical Micro- and Nanoswimmers
}

\section{Review Article}

\section{Author(s):}

Pané, Salvador; Puigmarti-Luis, Josep; Bergeles, Christos; Chen, Xiang-Zhong; Pellicer, Eva; Sort, Jordi; Počepcová, Vanda; Ferreira, Antoine; Nelson, Bradley J.

\section{Publication date:}

2019-04

\section{Permanent link:}

https://doi.org/10.3929/ethz-b-000335802

Rights / license:

In Copyright - Non-Commercial Use Permitted

\section{Originally published in:}

Advanced Materials Technologies 4(4), https://doi.org/10.1002/admt.201800575

\section{Funding acknowledgement:}

771565C - Highly Integrated Nanoscale Robots for Targeted Delivery to the Central Nervous System (EC)

160174 - Controlled Crystal Growth and Large Scale Integration of Functional Materials by Microfluidic Means (ColnFun) (SNF) 
DOI: $10.1002 /($ please add manuscript number $))$

Article type: Review

\section{Imaging Technologies for Biomedical Micro- and Nanoswimmers}

Salvador Pané*, Josep Puigmartí-Luis*, Christos Bergeles, Xiang-Zhong Chen, Eva Pellicer, Jordi Sort, Vanda Počepcová, Antoine Ferreira, Bradley J. Nelson

Dr. S. Pané, Dr. X.-Z. Chen, MUDr. V. Počepcová, Prof. B. J. Nelson

Multi-Scale Robotics Lab (MSRL), Institute of Robotics and Intelligent Systems (IRIS), ETH Zurich, Tannenstrasse 3, CH-8092 Zurich, Switzerland

E-mail: vidalp@ethz.ch

Dr. J. Puigmartí-Luis

Institute of Chemical and Bioengineering, ETH Zürich, Vladimir Prelog Weg 1, 8093 Zürich, Switzerland

E-mail: josep.puigmarti@ chem.ethz.ch

Prof. C. Bergeles

Robotics and Vision in Medicine Lab, School of Biomedical Engineering and Imaging Sciences, King's College London

Dr. E. Pellicer

Departament de Física, Facultat de Ciències, Universitat Autònoma de Barcelona, Campus UAB, 08193 Bellaterra, Spain

Prof. J. Sort

Institució Catalana de Recerca i Estudis Avançats (ICREA) and Departament de Física, Universitat Autònoma de Barcelona, 08193 Bellaterra, Spain

Prof. A. Ferreira

Institut National des Sciences Appliquees Centre Val de Loire, 88 boulevard Lahitolle, 18020 Bourges, France

Keywords: small-scale swimmers, optical tracking, magnetic resonance imaging, X-rays imaging, ultrasound, fluorescence functionalization 


\begin{abstract}
The last decade has seen the rapid development of untethered mobile micro- and nanorobots able to navigate liquids by means of external power sources or by harvesting chemicals from their surrounding media. These tiny devices hold great promise for applications in the biomedical field including targeted drug delivery, localized diagnostics, microsurgery, cell stimulation. However, to translate small-scale robots from the laboratory to the clinic, many challenges remain. A major obstacle is the lack of imaging technologies that will allow for precise tracking of the devices in vivo. Here we review current progress, challenges, and future possibilities in the monitoring and tracking of biomedical micro- and nanomachines using established as well as less conventional imaging technologies.
\end{abstract}




\section{Introduction}

Small-scale robots are miniaturized tetherless machines capable of navigating through different fluid environments by harvesting fuels from their surroundings,${ }^{[1]}$ by external energy sources, ${ }^{[2]}$ or powered by motile cells. ${ }^{[3]}$ Advances in micro- and nanomanufacturing, manipulation engineering, surface chemistry and catalysis have led to a new generation of biomedical micro and nanomachines with unprecedented capabilities. To date, micro- and nanorobots have demonstrated the capability to perform several tasks in the biomedical arena, such as targeted drug delivery, localized biopsy, bioanalysis, cell sorting, detoxification, and target isolation. ${ }^{[4]}$ While most early research using biomedical micro- and nanorobots has been realized in vitro, preliminary studies have recently demonstrated the potential of these machines in vivo. ${ }^{[5]}$ Despite these achievements, existing micro- and nanorobotic platforms require that a number of issues are further addressed in order to achieve for clinical translation. Some aspects requiring significant development include the integration of multiple building blocks, machine-to-machine communication, control and actuation over machine swarms, enhanced biocompatibility and biodegradability, and compartmentalization. One of the most critical obstacles for the translation of small-scale robotics to relevant clinical applications is tracking and monitoring the devices inside the body. Three-dimensional visual servoing of micro- and nanomachines is key for in vivo applications such as micro- and nanosurgery or supply of drugs and radioactive seeds to affected tissue. Currently, this can be wirelessly accomplished by means of three-dimensional (3D) navigation approaches (i.e., electromagnetic fields, acoustic waves or light). However, to precisely control the motion and actuation of small biomedical devices in vivo, real-time 3D tracking control is necessary.

Visual servoing of micro- and nanomachines requires a synergistic integration of several techniques such as imaging technology and image processing, control theory, and contrastagent functionalization, among others. Some conventional medical imaging techniques used for revealing the body internal organs and tissues have been tested to monitor the position of 
small-scale structures and devices. Nanotechnology and surface chemistry offers many options for the production of contrast agents, and it has already impacted the field of imaging technologies. However, it is not the aim of this review to cover research studies on small-scale particulate-based contrast agents. For these studies, the reader is referred to previous articles and reviews. ${ }^{[6]}$

The present contribution reviews current progress, challenges and future possibilities in the visual monitoring of biomedical micro- and nanomachines using established and less conventional imaging technologies.

\section{Imaging Technologies for Micro- and Nanoswimmers}

\subsection{Optical Tracking for microrobot localization}

The vast majority of experimental realizations of micro and nanorobots have occurred on the benchtop under well-defined experimental conditions. In such setups, the primary means of microrobot visualisation and localisation is through optical microscopy image capture and subsequent processing. The main challenges pertain to identifying multiple microdevices and independently tracking them, and extracting the full six degree-of-freedom pose of the device rather than only their planar coordinates. We note that particle tracking with optical microscopy is a very well researched topic with numerous contributions. ${ }^{[7]}$ Therefore, we would like to constrain our focus on research that has been applied in the domain of microand nanorobots.

Naturally, techniques for 2D and 3D microdevice tracking under optical microscopy rely on well calibrated imaging systems. Calibration of optical microscopes is an active research topic, with research contributions steadily updating the precision with which the image formation process is characterized. One of the early works in the domain ${ }^{[8]}$ introduced a parametric model of the optical microscope and derived the projection model using a single calibration pattern images perpendicularly to the optical axis. More recently, Luo et al. 
combined the Gauss lens model and projective camera model to perform parameter estimation by calibration patterns image at near parallel configurations. ${ }^{[9]}$ Further, Ammi et al. used a virtual $3 \mathrm{~d}$ calibration pattern which was created by moving the tip of a micromanipulator imaged with subpixel accuracy by an optical microscope. ${ }^{[10]}$ All these and similar approaches generally do not tackle the issue of aberrations, apart from radial and/or tangential distortions that have well defined mathematical models. More detailed approaches, such as methods for comprehensive aberration correction developed by Stavis and coworkers, boost the confidence in the measurements extracted by using optical microscopy systems. ${ }^{[1]}$

\subsubsection{Techniques for $2 D$ tracking}

Intensity-based trackers have been used in a variety of microrobotic applications and experiments, as microrobotic devices tend to be dark objects moving over a light or transparent background. Therefore, simple thresholding operations followed by morphological filters and connected component analysis have been shown robust in identifying multiple microdevices. Morphological filters like dilation and erosion achieve the removal of small blob/components that arise due to noise or workspace contamination. Connected component analysis then retrieves morphological characteristics of the segmented blobs (e.g. size, location, principle axes) - each connected component/blob represents a microdevice. Linking the extracted connected components through time allows the tracking of the microdevices (temporal segmentation). Using this technique, one could track artificial bacterial flagella and characterize their behavior. ${ }^{[12]}$ The orientation of the flagella was given by post-processing the blobs by line fitting. Similar algorithms have been applied for tracking elliptical microrobots moving within eight-coil Magnetic Field Generators (MFG) commercialized by MagnetbotiX AG (www.magnebotix.com) including the Octomag, ${ }^{[13]}$ Minimag, ${ }^{[14]}$ and Nanomag. ${ }^{[15]}$ Intensity-based tracking has also been employed to track microdevices in lapine eyes, in vivo. ${ }^{[16]}$ (Figure 1 (a), (b)) These intensity-based algorithms 
are also applicable for $\mu$ Bot, ${ }^{[17]}$ "micro-rockets", ${ }^{[18]}$ and, generally, for any microdevice that is

In microrobotic applications that involve rapidly but controllably moving devices, background-subtraction (motion-based) localization has proven beneficial. A preliminary step of motion detection allows the estimation and subsequent removal of the background, therefore removing noise arising from image capture and workspace contamination. Magmite, a microrobot based on wireless resonant magnetic micro-actuators, was tracked using a combination of background subtraction and intensity-based tracking, wherein the first seconds of Magmite motion serves to separate the microdevice from the background. ${ }^{[19]}$

Other approaches to microrobotic tracking involve colour-based probabilistic tracking, which are especially applicable in a medical context where intensity-based tracking and motion-based tracking underperform due to general motion of the anatomy, specular reflections, and lack of salient features that complicate the application of generic tracking algorithms. In ${ }^{[20]}$, a level-set tracker was applied to identify and follow microrobots that move near the retina of phantom eyes. Manually segmented images that indicated the microdevice and the background were used to train the colour profiles of both the microdevice and the retinal background. Subsequently, the algorithms could identify the locations within the image that, based on their colour, were determined to belong to the microdevice rather than the retinal background. This algorithm was amenable to straightforward improvements by incorporating shape information, therefore, further limiting the effect that noise and similar-coloured-regions have on microrobot detection.

\subsubsection{Techniques for $3 D$ tracking}

Building on the algorithms that achieve the detection and tracking of microdevices on 2D images, research has been conducted on extracting full 3D motion. In cases where a stereomicroscope is employed, common approaches involving feature extraction (e.g. entire blob 
detection), left-to-right image feature matching and subsequent triangulation, can recover the acquisition, making stereo-based 3D localisation impossible. In such cases, focus cues or CAD models of the object to be tracked are used to retrieve 3D information, even the full 3D pose (6 degrees-of-freedom) of the microdevice.

Depth-from-focus/defocus retrieves the third spatial dimension through the examination of focus/defocus cues after the planar coordinates of the microdevice have been extracted. ${ }^{[21]}$ The fundamental assumption is that defocus of a microdevice creates a "halo" that can be approximated as the convolution of a Gaussian-blur kernel with the ideal and sharp image of the microdevice. The size of the "halo" is related to the distance of the microdevice away from the in-focus plane. Therefore, depth-from-defocus algorithms estimate the size of the Gaussian-blur kernel, and, then, the depth of the device using thin-lens equations that relate the blur with the in-focus plane. Similarly, depth-from-focus is based on the assumption that in a calibrated optical system, the exact location of the in-focus plane is known. Therefore, if microdevices can be retained (or brought) in focus, their depth will be known. This approach is simpler to implement in practice, especially since most micromanipulation workspaces are equipped with motorised microscopes. Depth-from-focus, therefore, was used to retain a microdevice in focus and estimate its dept. ${ }^{[22]}$ In a more complex imaging system involving non-linear optics, raytracing, and focus was used to estimate the depth of an intraocularly moving bead. ${ }^{[23]}$

Full six degree-of-freedom localisation has seen limited applications in microdevice manipulation, primarily because most efforts in microrobots are focused on developing the microdevices, functionalising them, and conceiving micromanipulation systems. However, work from the intraocular microrobotics domain employs a CAD model of the microdevice to perform pose estimation. The CAD model of the microdevice is rotated and translated so that its virtual projection (using a camera calibration matrix) on the capture images matches the 
microdevice detected in $2 \mathrm{D} .{ }^{[24]}$ The ambiguity of perspective projection is addressed using the metric model of the object of interest inspired by seminal work in computer vision. ${ }^{[25]}$

\subsection{Magnetic imaging}

\subsubsection{Magnetic resonance imaging}

Magnetic resonance imaging (MRI) was developed during the 1970s-1980s as a medical imaging technique that enables the acquisition of images from biological tissue in a noninvasive and precise manner due to excellent tissue contrast and spatial resolution. MRI is based on a phenomenon known as nuclear magnetic resonance (NMR), which utilizes strong magnetic fields in combination with radiofrequency (RF) electromagnetic waves and magnetic field gradients. ${ }^{[26]}$ More specifically, NMR is based on the ability of atomic nuclei of interest to absorb and re-emit electromagnetic radiation when placed in the presence of a constant magnetic field. First, a strong magnetic field is used to align the magnetic nuclear spins; then, a perturbation to this alignment is induced by a RF pulse. By properly tuning the frequency of the electromagnetic pulse (which in turn depends on the strength of the applied magnetic field) the nuclear spins undergo a resonant absorption and subsequently relax, emitting energy that can be measured and processed in order to yield an NMR spectrum. In this way, important physical, chemical, electronic and structural information about molecules in a solution or in the solid state (their topology, dynamics and 3D structure, for example) can be obtained. In clinical MRI, hydrogen nuclei (protons) are those used for imaging since they exist abundantly in the human body, particularly in fat and water. By applying different types of RF pulses under the magnetic field, different contrasts can be generated based on the relaxation of protons at different locations. The magnetic field gradients are used to precisely locate the signal in the 3D space. MRI surpasses the versatility of other imaging techniques, such as X-ray fluoroscopy, in the sense that it can be used to readily acquire 3D images rather 
than 2D plane projections (as in the case of fluoroscopy), while also circumventing the

The principle of MRI image generation relies on the application of a perfectly homogeneous magnetic field together with precisely controlled superimposed field gradients. If magnetic objects are introduced in the body, e.g., untethered micro- and nanorobots, their magnetic susceptibility causes important artifacts, i.e., distortions, in the resulting images. ${ }^{[27]}$ An additional challenge is the minimization of image acquisition delays for real-time applications, as reported by O. Felfoul et al. ${ }^{[28]}$ A. Ferreira and collaborators ${ }^{[29]}$ showed that with a thorough understanding of the process of MRI artifact formation from a magnetic object, one can engineer image-processing algorithms that allow for precisely positioning magnetic materials in MRI scans (Figure 1 (c)). In this way, MRI offers a unique integrated environment platform for the tracking and navigation of ferromagnetic devices, such as magnetic carriers for targeted drug delivery, implantable biosensors, and even surgical microtools. ${ }^{[28-29]}$ Observability of microagents in MRI is limited by spatial resolution of existing medical scanners. However, navigable magnetic microswimmers much smaller than a single MRI voxel (500 $\mu \mathrm{m}$ for clinical scanners) can be synthesized in order to exhibit a magnetic signature much larger than the microswimmer itself. ${ }^{[30]}$

MRI for in vivo tracking magnetic helical microrobots inside rodent stomachs has been recently exploited by Zhang and co-workers. ${ }^{[31]}$ The micromachines were fabricated by coating Spirulina, microalgae with a helical shape, with magnetite films. Figure 1 (d) shows swarms of helical microrobots inside the stomach of rats. The same authors successfully demonstrated that these microrobots can also be imaged in vivo in the subcutaneous tissue and in the intraperitoneal cavity of mice (see the section "Fluorescence imaging").

MRI can also be employed as a means to wirelessly guide and manipulate magnetic micro- and nano-objects inside the body, taking advantage of the magnetic field gradients typically utilized for the imaging purposes, as recently reported by K. Belharet et al., ${ }^{[32]}$ Kosa 
et al., ${ }^{[33]}$ and S. Martel et al. ${ }^{[34]}$ This is appealing since the complete system benefits from the hardware (three orthogonal coils are assembled to generate precise magnetic field gradients in clinical MRI) and software scanner facilities already available in many hospitals. By using MRI for both tracking and propulsion of untethered micro- and nanorobotic platforms, ${ }^{[35]}$ one can engineer a computer-controlled close-loop navigation system [36] that may create opportunities for new therapies, such as micro-surgery at remote locations within the body with little trauma to healthy tissue or targeted drug delivery.

While this is exciting, the approach faces various challenges ${ }^{[32]}$ including the following: (i) different magnetic field gradients are needed for imaging and guiding purposes, hence a time-dependent multiplexed sequence is necessary, (ii) pulsatile flows that vary in waveform, amplitude and frequency exist in the different vessels and this variation must be taken into account, (iii) magnetic field gradients in conventional MRI devices are limited in amplitude, (iv) delays between image processing and actuation, which can result in unstable navigation control, should be minimized using appropriate algorithms, (v) overheating in MRI due to radiofrequency resonance heating, ${ }^{[3]}$ that can pose limitations in the duty cycle, should be avoided; (vi) while MRI systems can resolve features down to $100 \mu \mathrm{m}$, the acquisition time is on the order of seconds, which challenges the traceability of micro- and nanomachines; furthermore, resolution significantly degrades when imaging speed increases.

Multi-imaging modalities such as combining X-ray with MRI can also be considered. The main problem caused by motion artifacts degrading navigation control can be improved by combining X-ray platforms including computer-tomography (CT) for real-time tracking error regulation. ${ }^{[38]}$ Progress in MRI research has been tremendous in recent years, feedback control units have been significantly improved, and MRI has become one of the most appealing methods not only for medical imaging but also as means for targeted minimally invasive therapies. ${ }^{[39]}$

\subsubsection{Magnetic particle imaging}


Magnetic particle imaging (MPI) is an emerging imaging technique that can directly visualize the position and concentration of superparamagnetic nanoparticles (SPMNPs). It was first conceived in 2001 and proposed by Gleich and Weizenecker in 2005 at the Philips Research Laboratory in Hamburg, Germany. ${ }^{[40]}$ SPMNPs exhibit a nonlinear magnetization curve. When an alternating magnetic field is applied on the SPMNPs, the modulation of the magnetization causes an induction of an electric signal that can be received by electromagnetic pickup coils. Such a signal can only be generated by SPMNPs, as biological tissue is diamagnetic and it does not generate any interfering signal. Compared with MRI, which usually needs a field of several tesla to excite the protons, the MPI usually just requires a few tens of millitesla because the SPMNPs has $10^{8}$ times higher magnetization and $10^{4}$ times faster relaxation. ${ }^{[41]}$ These characteristics lead to a superb spatial resolution (up to $1 \mathrm{~mm}$ ) as well as high acquisition rates of up to 40 volumes per second. ${ }^{[40 b, 42]}$

Currently, commercially available MPI systems are designed for research in small animals such as mice and rats. The main technical challenge is how to upscale the system to humansize by considering both the frequency and the amplitude of the oscillating magnetic fields that are used for the signal generation. ${ }^{[40 c]}$ As MPI is a relatively new imaging technique, we could not find any work reporting the use of this technique for imaging micro- and nanorobots. Nevertheless, we can foresee the potential of MPI as a powerful tool for in-vivo tracking of micro- and nanorobots.

\section{$2.3 X$-ray imaging}

X-ray imaging exploits the differential absorption or scattering of X-ray beams passing through the human body in order to reveal its internal structures. The most common techniques include computed tomography (CT), radiography, and fluoroscopy. Among them, fluoroscopy is the most promising approach to perform tracking of small-scale swimmers as it allows real-time acquisition of images up to $30 \mathrm{fps}$. 
Fluoroscopy, also known as fluoroservoing, is a dynamic real-time imaging method pacemakers, or the use of catheters. In the vast majority of cases, iodine-based contrast agents are used to enhance the contrast of the region of interest (i.e. vessel, parts of the gastrointestinal track). Pioneering studies using X-ray fluoroscopy in medical robotics include needle-based treatments by Navab et al. and Loser et al., ${ }^{[43]}$ in which fluoroscopy was employed to control the orientation of needles. For more details on this research, we refer the reader to the review paper by Patel and co-workers. ${ }^{[4]}$ As fluoroscopy can distinguish between materials that differ in density by less than $1 \%$, this technique is also useful to track metallic structures. However, little work exists regarding the use of fluoroscopy for monitoring the activity of micro- and nanorobots. Park's group proposed a magnetic millirobot for therapies addressing chronic total occlusions. ${ }^{[45]}$ The device consisted of a neodymium-iron-boron magnet with a length of $10 \mathrm{~mm}$ and a diameter of $1 \mathrm{~mm}$. An electromagnetic system was used to guide the microrobot through the aorta of a living rabbit, while the tracking was performed using X-rays. Figure 2 (a) shows a sequence of X-ray images showing a microrobot travelling against the blood flow from the kidney branch to the aortic branch. The same group recently reported a therapeutic intravascular magnetic microrobot, which is maneuvered using an electromagnetic system consisting of a combination of Helmholtz, Maxwell, rotational saddle and gradient saddle coils. ${ }^{[46]}$ Locomotion and hammering tests in blood vessels were realized in vivo with mini-pig animal models. The microrobot consisted of a 10-mm-long neodymium iron-boron cylinder with a diameter of $1 \mathrm{~mm}$ and pointed ends. An X-ray fluoroscope was used to track the position and movements of the magnetic microrobot. Figure 2 (b) shows a superposed X-ray image sequence of an in vivo experiment of the proposed microrobot propelling and hammering in the abdominal aorta of a mini-pig. Kim and co-workers have recently developed a bi-planar X-ray imaging system for real-time position recognition of magnetic microdevices. ${ }^{[47]}$ 
However, the system was only evaluated using a phantom. Figure 2 (c) shows the proposed biplanar X-ray imaging setup integrated in an electromagnetic system. Recently, Nelson and co-workers proposed a method for tracking magnetically guided catheters in three dimensions with a single rotating C-Arm fluoroscope ${ }^{[48]}$ A comparable level of position accuracy as in biplanar fluoroscopy can be reached with this method. Additionally, the space required by the imaging system in the operating room can be minimized using this approach.

CT is an X-ray imaging technique that exploits the tomographic reconstruction of body cross-sectional X-ray images obtained from different angles. CT can also be combined with other imaging techniques such as positron emission tomography (PET), ${ }^{[49]}$ or single photon emission computed tomography (SPECT). ${ }^{[50]}$ An interesting approach for tracking microrobots was recently explored by Sanchez and co-workers, which demonstrated that CT combined with PET can be used to track a swarm of catalytic micromotors. ${ }^{[51]}$ PET is an imaging technique, which is based on the detection of gamma rays that are emitted when a positron released by the radionuclide collides with an electron of the surrounding matter (i.e. body tissue). The group of Sanchez evaluated this technique to track the motion of catalytic tubular microswimmers in a phantom. The microswimmers comprised a poly-(3,4ethylenedioxythiophene) (PEDOT) outer layer, and an inner layer of platinum. The last layer serves as the catalyst for the decomposition of hydrogen peroxide in the inner cavity of the tubular structures. As a result of the decomposition of $\mathrm{H}_{2} \mathrm{O}_{2}$, oxygen bubbles are formed and ultimately impart momentum to the structures, leading to their propulsion. The PEDOT layer was coated with evaporated gold, which allowed for the chemisorption of the radiotracer iodine-124 $\left({ }^{124}\right.$ I). Figure 3 shows the optical, SEM and STEM images of the micromotors, a scheme showing their functionalization with ${ }^{124} \mathrm{I}$, and PET-CT images of a swarm of catalytic microswimmers moving through a phantom channel obtained at different time ranges.

While X-ray imaging has been employed for tracking miniaturized robotic devices, this technique has not been sufficiently investigated for monitoring micro- and nanoswimmers 
most probably due to its low spatial resolution. Focal spot sizes in fluoroscopic tubes can be as small as $0.3 \mathrm{~mm}$. Nevertheless, current developments in nanoparticulate-based contrast agents will impact research in micro- and nanorobotics. Additionally, the incorporation of high-speed cameras together with advanced computer-controlled tracking systems will facilitate the tracking of small devices without limiting their motion capabilities. ${ }^{[52]}$

\subsection{Ultrasound imaging}

Ultrasound (US) or ultrasonic imaging is a noninvasive, versatile and well-established technique that is regularly used in medical settings. This technique is based on the reflected acoustic waves that have interacted with body tissues. ${ }^{[53]}$ When ultrasonic waves encounter an interface between two mediums with different acoustic properties, the waves are partially scattered, partially reflected or absorbed. The more different the acoustic properties of two interfacing tissues are, the higher the intensity of the reflected acoustic waves is. Compared to other imaging techniques such as magnetic resonance imaging (MRI), fluoroscopy, computed tomography (CT), and positron emission tomography (PET), ultrasound combines high temporal (real-time control) and spatial resolution with minimum adverse health effects and at a lower cost. However, there are numerous drawbacks associated with US, such as operator inter-variability, limited scanned area of the patient, low signal-to-noise ratio, and strong scattering from bones and air pockets. US-based imaging techniques can be partially overcome through passive localization (passive mode), in which an ultrasonic signal of a very specific pattern is generated by a microrobot. ${ }^{[54]}$

Ultrasound imaging systems operate at frequencies between 1 and $100 \mathrm{MHz}$ and provide a spatial resolution of a millimetre to micrometers. Ultrasound is a particularly attractive since the method is used to penetrate the human body for scanning at different depths applying various frequencies. For example, a $1 \mathrm{MHz}$ ultrasound penetrates the body to $4 \mathrm{~cm}$ in muscle and $15 \mathrm{~cm}$ in fat, respectively. US offers opportunities to visualize human tissue and provide 
visual feedback for microrobot control when injected into a living body. Recently, ultrasound as the paramagnetic particles, ${ }^{[55]}$ magnetotactic bacteria, ${ }^{[56]}$ self-propelled microjets, ${ }^{[57]}$ and bio-inspired magnetosperm ${ }^{[58]}$ in living tissue. Though, the spatial resolution of the US is acceptable, there are uncertainties associated with the position of microrobots. The positioning error arises due to the inaccuracy of the US-based visual feedback, which is provided by the tracking algorithm. Additionally, accurate position estimation of the microrobot is compromised due to imaging artifacts, the presence of "disturbance" objects, and weak contrast with the image background.

Recently, Misra's group demonstrated wireless motion control of magnetic particles using an ultrasound feedback system. ${ }^{[55]}$ The particles were manipulated using magnetic field gradients along an S-curve. The position of the microparticles was first determined from the ultrasound images. The calibration of tracking of microparticles was validated using a calibrated microscopic system. Finally, the feedback provided by the US system was used to implement a proportional-derivative magnetic-based control system. Using this approach, the authors achieved point-to-point control of the microparticles with an average position tracking error and average speed of $48 \mathrm{~mm} / \mathrm{s}$ and $191 \mathrm{~mm} / \mathrm{s}$, respectively. In another study, Misra and co-workers determined the position of the self-propelled microjet using a pulse-echo approach with a brightness-mode (B-mode) US display. ${ }^{[59]}$ The microjets were magnetically steered using two air-core coils capable of generating magnetic torques within a plane. The binary image analysis provided accurate control of the microjets that is moving at an average velocity of $156 \pm 35.1 \mu \mathrm{m} / \mathrm{s}$ and an average tracking error of $250.7 \pm 164.7 \mu \mathrm{m} .{ }^{[57]}$ More recent work from the same group use feedback extracted from ultrasound images to realize wireless magnetic motion control of a hydrogel-based gripper (Figure 4 (a)). ${ }^{[60]}$

The localization of a wireless microrobot can also be accomplished in passive US mode if the microrobot acts as an emitter ${ }^{[61]}$ by incorporating an ultrasound transducer (e.g. a 
cantilever) in the microrobot architecture. In such a case, the signal emitted by the transducer principle, since the ultrasonic signals only need to travel through the media once, this approach offers twice the penetration depth than the active mode. Probst, Flückiger et al. showed the manufacturing of a hybrid acoustic microrobotic transmitter by means of microfabrication (photolithography, electrodeposition, selective etching) and robotic microassembly. ${ }^{[62]}$ The transmistters consisted of two gold architectures separated by a polymeric spacer: (a) a bottom gold layer containing a magnetic micropart in the center; and (b) a gold structure comprising two beam springs, which were used to hold a magnetic microstructure in the center (Figure 4 (b)). By applying an oscillating magnetic field, the unthethered resonator exhibited a wireless acoustic emission in the range of $\mathrm{kHz}$. The passive US mode was also exploited in antibody conjugated magnetic nanoparticles for tumor localization. Following nanoparticle injection and their aggregation at the tumor site, a harmonic magnetic field of tens of $\mathrm{kHz}$ was applied to vibrate the MNP-tumor complex and generate acoustic waves detectable on the patient's surface by an acoustic sensor. ${ }^{[63]}$ Through this approach, information on tumor presence a few $\mathrm{cm}$ deep under the tissue surface was achieved. An algorithm was later developed by the same authors to localize the tumor in 3-D in real time. ${ }^{[64]}$ The fabrication and controlled navigation of nanorobots with an onboard ultrasound transducer will likely deeply be exploited in the immediate future.

Recent examples in the literature demonstrate the versatility of US in providing locomotion. For example, the fabrication and performance of US-propelled magneticallyguided Au-Ni-Au magnetic nanowires were recently shown by Wang's group. ${ }^{[65]}$ In this work, ultrasound waves were produced by an external piezoelectric transducer. The motion of the nanomotors was visualized optically using a CCD camera. Following the same approach, the authors employed US signals to control the movement of bubble-propelled chemically powered PEDOT/Ni/Pt micromotors. ${ }^{[66]}$ Recently, Ahmed and coworkers developed a hybrid 
acousto-magnetic soft microrobot consisting of a microbubble trapped in a superparamagnetic

polymer composite. ${ }^{[67]}$ The trapped microbubble enables propulsion in an external acoustic field, and the magnetic particles allow controlled motion in a magnetic field. The authors used this method to manipulate the swimmer along a path to write "ETH". This approach can be used to maneuver swimmers in a three-dimensional environment. Stavis and co-workers measured the microvortical flows around gold nanorods propelled by ultrasound in water using polystyrene nanoparticles as optical tracers. Their results presented an important step towards understanding the hydrodynamics of the interaction between ultrasound stimulated particles and biological media. ${ }^{[68]}$ The combination of US for both propulsion and tracking is an obvious next step.

\subsection{Fluorescence imaging}

Among the various strategies employed to track synthetic micro- and nanoswimmers, fluorescence imaging is the most preferred approach for rapid and real-time in vitro investigations. New advances made in the design of fluorophore moieties and easy functionalization of the surface of micro- and nanoswimmers are assets that have further aided the prevalence of fluorescence microscopy in this particular research field. Likewise, important advances in nanofabrication strategies and the increasing number of materials used to design novel self-propelled micro- and nanostructures have aided a number of synthetic routes employed to append a large variety of fluorescent and biomolecular dyes on their surface. Precise monitoring of the spatial location and motion of micro- and nanoswimmers is necessary to address practical and real-life applications in complex biological conditions, such as targeted drug delivery or diagnostic applications at specific locations inside the human body. In this section, we give a comprehensive review of the most recent strategies being used to derivatize and visualize the motion (and position) of micro- and nanoswimmers in different surroundings. Tailored chemical design of the surface of self-propelled structures can 
facilitate specific binding events and, hence, can lead to custom functionalization for specific tasks and applications.

\subsubsection{Surface chemical functionalization of self-propelled micro- and nanostructures}

The surface chemistry of micro- and nanoswimmers is based on the materials used in their fabrication. In most reported research, carboxylic acids or amine groups present on the surface of self-propelled structures are used as reactive sites to anchor fluorescent moieties and biomolecule-based dyes. As shown in Figure 5, surfaces with carboxylic acid termination groups can be chemically modified to amine-coated surfaces following a synthetic route that starts with the reaction of 1-ethyl-3-(dimethylaminopropyl)carbodiimide (EDC) with the carboxylic acid groups, followed by a reaction with $\mathrm{N}$-hydroxysuccinimide (NHS). ${ }^{[69]}$ Diamine-based moieties can be used in a subsequent reaction step to definitely achieve aminederivatized substrates (Figure 5 (a)i). On the other hand, amine-coated substrates can easily be converted to carboxylic acid activated surfaces with succinic anhydride (SA), see Figure 5 (b). ${ }^{[70]}$ In addition, both carboxylic and amine-coated substrates can append moieties bearing amine groups following the reaction routes shown in Figure 5 (a)ii and Figure 5 (b)i, respectively. Other synthetic routes can also be considered to functionalize carboxylic acid coated surfaces with amine containing ligands, which require, for example, the formation of corresponding acyl chloride. ${ }^{[71]}$

Molecules or surfaces bearing amine groups can be covalently linked to ligands (or surfaces) containing isothiocyanate (Figure 5 (c) ${ }^{[72]}$ or hydroxide groups once the amines are modified with succinimidyl iodoacetate (SIA), see Figure 5 (d)i). In this case, ligands bearing sulfhydryl groups can also be anchored to amine-coated surfaces following the same reaction mechanism (Figure 5 (d)ii ).

Strategies use to coat the surface of self-propelled micro- and nanostructures such as sputtering of metallic thin films or layer-by-layer deposition approaches employing oppositely 
charged polymers ${ }^{[73]}$ have also been reported in order to create functionalized surfaces that can target and append organic and biomolecular fluorophores. Metals such as gold, for example, can be easily coated with thiolated molecules bearing carboxylic acid and amine groups employing self-assembled monolayer (SAM) chemistry (Figure 5 (e)), ${ }^{[74]}$ whereas other metals such as titanium must be anodized to generate an oxide layer that can further be coated with silane molecules or ligands containing isothiocyanates, see Figure 5 (f)i and Figure 5(f)ii, respectively. ${ }^{[75]}$ Silica coatings are also widespread in the field where functionalization with silane moieties incorporating carboxylic or amine groups is straightforward after surface hydrolyzation. ${ }^{[76]}$ It should be noted that the derivatization of gold, titanium and silica with amine or carboxylic acid groups eases further functionalization strategies with other moieties following the reaction routes presented in Figure 5 (a) - (d).

Recently, bioorthogonal chemistry has been widely adopted to label biochemical reactions in biologically relevant environment, especially in living systems. Bioorthogonal reactant pairs are molecular groups that are mutually reactive but do not cross-react or interact with biological functionalities or reactions in a cell. The reaction between the reactant pair is highly specific and fast, and their products are stable and nontoxic. Besides, the bioorthogonal chemical reactions usually take place at relatively mild conditions, e.g. in water at room temperature. Due to these advantages, the biorthogonal chemical reactions are promising for efficient and site-specific surface modification. For example, the Staudinger-inspired reaction between azides and phosphines to yield amide linkages developed by Bertozzi and coworkers is now a classic among bioorthogonal processes. ${ }^{[77]}$ To date, numerous bioorthogonal reactant pairs have been discovered. For more information on bioorthogonal chemistry, the readers are referred to some reviews in this field. ${ }^{[78]}$

\subsubsection{Mobile micro- and nanostructures functionalized with fluorophores}


Recently, Maier et al. have presented a bio-labelling strategy to track the motion of magnetically propelled DNA-based microstructures by chemically tagging some of the constituent DNA strands with a cyanine dye (Cy3) on their $5^{\prime}$ ends. ${ }^{[79]} \mathrm{Cy} 3$ is a fluorescent molecule frequently used to chemically label nucleic acids and has an excitation wavelength located at around $550 \mathrm{~nm}$ and an emission peak at $570 \mathrm{~nm}$ (Figure 6 (a)). In this work, Cy3DNA strand fluorophores located on the surface of spherical magnetic beads (approx. $1 \mu \mathrm{m}$ in diameter) facilitated a thorough characterization of the motion style of these self-propelled hybrid structures. With the use of fluorescence microscopy, the authors concluded that the DNA fragments anchored on the surface of the magnetic beads were responsible for the movement. They demonstrated that the DNA fragments operated as flagella bundles when external rotating magnetic fields were applied (Figure 6 (b)). Clearly, this biomolecular fluorophore labeling strategy not only enabled the visualization of the movement of the hybrid particles in in vitro experiments but also confirmed that these structures resemble the motion of self-propelling flagellated bacteria found in nature (Figure 6 (c)). Recently, Hoop et al. developed a smart multifunctional drug delivery nanoplatform for targeting cancer cells that was bio-labeled. ${ }^{[80]}$ In this work, the nanomachine consisted of a magnetic tube with a $\mathrm{pH}-$ responsive chitosan hydrogel in its inner cavity. The outside of the tube was coated with gold and fluorescein isothiocyanate (FITC, Figure 6 (d)) labeled thiol-ssDNA, a biological marker, was conjugated on its surface by thiol-gold click chemistry (Figure 5 (e)), which enabled traceability under a fluorescent microscope (Figure 6 (e) and (f)).

Other researchers have also shown that surface functionalization of micro- and nanoswimmers with fluorescent active moieties not only enables monitoring of their motion but also new and rapid analytic methods. In this context, ultrasound driven nanowires functionalized with biomolecular fluorophores (dye-labelled single-stranded DNA) have, for example, been used to screen cancer cells in vitro. ${ }^{[81]}$ In this study, graphene-oxide (GO) coated nanoswimmers were used to quench the fluorescence signal of dye-labeled DNA 
strands that can recover their fluorescence upon hybridization with targeted biomolecules

\subsubsection{In vivo tracking of mobile micro- and nanostructures functionalized with fluorophores}

Conventional organic and biomolecular fluorophores can only be used for direct visualization experiments, a result that excludes these moieties from practical in vivo applications. ${ }^{[84]}$ Consequently, particular attention should be given to the functionalization of self-propelled micro- and nanostructures with probes that can respond to wavelengths above $600 \mathrm{~nm}$, i.e. in the near-infrared region (NIR) of the electromagnetic spectrum $(600-900 \mathrm{~nm})$ where tissue absorption is low and autofluorescence is negligible. ${ }^{[85]}$ Indeed, NIR dyes can generate visible emission upon excitation with a laser source operating in the near-infrared region (NIR), which facilitates in deep-tissue optical imaging. ${ }^{[86]}$

Accordingly, the need for biocompatible labelling systems to track in vivo motion of selfpropelled micro- and nanostructures has encouraged new roadmaps for in vivo fluorescence imaging in the near-infrared (NIR) spectral range. For example, this has been the focus of a recent work by Nelson and co-workers, where labelling of wirelessly controlled magnetic 
microswimmers with a helical shape, known as artificial bacterial flagella (ABFs), has been noted here that the number of NIR-797 molecules on the surface of ABFs was low in these investigations because the reactivity of the isothiocyanate group present in the NIR fluorophore with the hydroxyl groups on the hydrolysed titanium surface of ABFs is conformationally hindered (Figure 7 (b)). The yield of NIR-797 molecules on final structures was calculated to be around 5\%. The reaction used in this study is also summarized in Figure 5 (f, ii). ABF translational motion was achieved through body rotation in the presence of an external rotating magnetic field (Figure 7 (c)). With this functionalization strategy, the authors demonstrated, for the first time, in vivo tracking of magnetically controlled ABFs in the peritoneal cavity of a mouse (Figure $7(\mathrm{~d}, \mathrm{e})$ ). The functionalized ABFs could be precisely moved, monitored in real time, and their motion could be quantified employing weak rotating magnetic fields $(<10 \mathrm{mT})$. The velocity determined for the functionalized ABFs in in vivo studies was moderate and slower than the speed measured in in vitro experiments, which could be explained, among other factors, to the increased viscosity of the fluid present inside the intraperitoneal cavity.

In this study NIR 797, an isothiocyanate dye, proved to be efficient for in vivo imaging of self-propelled structures. Nonetheless, it should be considered that the absorption and emission peaks of NIR 797 are located at around $795 \mathrm{~nm}$ and $814 \mathrm{~nm}$, respectively. These wavelengths, as previously indicated, are transparent to tissue chromophores but the Stokes shift between the excitation and emission spectra is less than $30 \mathrm{~nm}$. This important overlap of the excitation and emission spectra of NIR 797 can, therefore, cause severe problems for deep-tissue optical imaging. ${ }^{[88]}$ It is for this reason that new imaging modalities for deeptissue imaging must be considered. A possible landmark in the field could be to develop multi-modal self-propelled micro- and nanostructures through the incorporation of different active moieties operating with other imaging technologies (e.g. radioactive agents). For 
example, Zhang and co-workers used magnetite-coated Spirulina-based microswimmers,

which not only could be tracked using MRI due to the intrinsic MRI contrast of magnetite, but also with fluorescence. ${ }^{[31]}$ Figure 8 shows fluorescence-based in vivo imaging of the swimmers in the subcutaneous tissue and the intraperitoneal cavity of nude mice.

Indeed, novel imaging scenarios such as photoacoustic (PA) imaging could also be combined and explored in this field of research. ${ }^{[89]}$

\section{Conclusions and perspectives}

While much progress has been made in developing synthetic micro- and nanoswimmers, ${ }^{[90]}$ the lack of in vivo imaging approaches is currently impeding the translation of these devices into clinical applications. ${ }^{[91]}$ Besides, in vivo environments are complex heterogeneous and dynamic systems, abundant in biomechanical forces (i.e.: body motion, lung expansion, heart beating, blood flow) ${ }^{\left[{ }^{[92]}\right.}$ The precise locomotion of micro- and nanoswimmers in such dynamic and complex environment, and consequently, their tracking will be very challenging. ${ }^{[93]}$ Therefore, efforts must be directed toward developing new imaging tools for self-propelled micro- and nanostructures that would allow for precise control of their localization and orientation in biologically relevant environments. This will open countless applications in medicine including targeted loading, transporting, biomedical imaging, tissue diagnosis, and drug delivery at specific areas of the human body with currently restricted accessibility.

The advantages and limitations of each imaging modality are summarized in Table 1 . It can be seen that there is no "one-for-all" imaging technique. Magnetic- and X-ray based imaging techniques have a large penetration depth in biological tissues. However, both of them require high-cost instrumentation and suffer from limited spatial resolution. Besides, $\mathrm{X}$ ray may also cause damage to biological tissues due to potential ionization. MRI is more biocompatible, but the real-time MRI imaging is still challenging. Ultrasound and optical 
imaging method including fluorescence-based optical tracking are relatively safe, and have high resolution, but the penetration depth of human tissue is low, which limits their application in certain circumstances. Microrobots with the size of a few mm or a swarm of nanorobots with similar swarm size should be relatively easy for imaging and tracking with the above-mentioned techniques. However, for tracking a single small-scale robot, an effective way for imaging and tracking with high resolution remains undeveloped. In all, different imaging techniques should be selected according to the specific imaging requirement, type of microrobots, and specific disease and situation of the patient. As suggested in ${ }^{[91]}$, a combination of technologies such as multispectral optoacoustic tomography seems the most promising path to move micro- and nanorobots in our bodies. Meanwhile, improvement in the resolution of the imaging techniques and enhancement in the contrast of the micro- and nanorobots to distinguish them with the surrounding environments should also be taken into consideration in the future.

\section{Acknowledgements}

Salvador Pané acknowledges funding from a Consolidator Grant from the European Research Council (ERC) under the European Union's Horizon 2020 research and innovation programme (grant agreement No. 771565). We also acknowledge the SBFI Cost Project No. C16.0061 under the COST Action MP1407. Christos Bergeles was supported by an ERC Starting Grant (714562), and a Wellcome/ESPRC Centre of Excellence Award [203145Z/16/Z]. J. P. L acknowledges founding from Swiss National Science Foundation (SNF) through the project no. 200021_160174 and the EU (ERC-2015-STG microCrysFact 677020). 
Received: ((will be filled in by the editorial staff))

Revised: ((will be filled in by the editorial staff))

Published online: ((will be filled in by the editorial staff))

\section{References}

[1] a) J. Katuri, X. Ma, M. M. Stanton, S. Sánchez, Acc. Chem. Res. 2017, 50, 2; b) H. Wang, M. Pumera, Chem. Rev. 2015, 115, 8704; c) J. G. S. Moo, M. Pumera, Chem. Eur. J. 2015, 21, 58; d) J. Parmar, X. Ma, J. Katuri, J. Simmchen, M. M. Stanton, C. Trichet-Paredes, L. Soler, S. Sanchez, Sci. Tech. Adv. Mater. 2015, 16, 014802.

[2] a) T. Xu, W. Gao, L. P. Xu, X. Zhang, S. Wang, Adv. Mater. 2017, 29, 1603250; b) X.-Z. Chen, M. Hoop, F. Mushtaq, E. Siringil, C. Hu, B. J. Nelson, S. Pané, Appl. Mater. Today 2017, 9, 37; c) X.-Z. Chen, M. Hoop, N. Shamsudhin, T. Huang, B. Ozkale, Q. Li, E. Siringil, F. Mushtaq, L. Di Tizio, B. J. Nelson, S. Pané, Adv. Mater. 2017, 29, 1605458; d) X.-Z. Chen, B. Jang, D. Ahmed, C. Hu, C. De Marco, M. Hoop, F. Mushtaq, B. J. Nelson, S. Pane, Adv. Mater. 2018, 30, 1705061.

[3] a) Z. Hosseinidoust, B. Mostaghaci, O. Yasa, B. W. Park, A. V. Singh, M. Sitti, Adv. Drug Deliv. Rev. 2016, 106, 27; b) V. Magdanz, M. Medina-Sanchez, L. Schwarz, H. Xu, J. Elgeti, O. G. Schmidt, Adv. Mater. 2017, 29, 1606301; c) M. M. Stanton, J. Simmchen, X. Ma, A. Miguel-López, S. Sánchez, Adv. Mater. Interfaces 2016, 3, 1500505; d) L. Schwarz, M. Medina-Sánchez, O. G. Schmidt, Appl. Phys. Rev. 2017, 4, 031301; e) M. M. Stanton, B. W. Park, A. Miguel-Lopez, X. Ma, M. Sitti, S. Sanchez, Small 2017, 13, 1603679.

[4] a) M. Sitti, H. Ceylan, W. Hu, J. Giltinan, M. Turan, S. Yim, E. Diller, Proc. IEEE 2015, 103, 205; b) J. Li, B. Esteban-Fernández de Ávila, W. Gao, L. Zhang, J. Wang, Sci. Robotics 2017, 2, eaam6431; c) B. J. Nelson, I. K. Kaliakatsos, J. J. Abbott, in Annu. Rev. Biomed. Eng., Vol. 12 (Eds: M. L. Yarmush, J. S. Duncan, M. L. Gray), 2010, 55; d) M. Hoop, A. S. Ribeiro, D. Rösch, P. Weinand, N. Mendes, F. Mushtaq, X.-Z. Chen, Y. Shen, C. 
F. Pujante, J. Puigmartí-Luis, J. Paredes, B. J. Nelson, A. P. Pêgo, S. Pané, Adv. Funct. Mater. 2018, 28, 1705920; e) X. Wang, X.-H. Qin, C. Hu, A. Terzopoulou, X.-Z. Chen, T.-Y. Huang, K. Maniura-Weber, S. Pané, B. J. Nelson, Adv. Funct. Mater. 2018, 1804107; f) X. Wang, C. Hu, L. Schurz, C. De Marco, X.-Z. Chen, S. Pane, B. J. Nelson, ACS Nano 2018, 12, $6210-6217$.

[5] a) W. Gao, R. Dong, S. Thamphiwatana, J. Li, W. Gao, L. Zhang, J. Wang, ACS Nano 2015, 9, 117; b) A. Servant, F. Qiu, M. Mazza, K. Kostarelos, B. J. Nelson, Adv. Mater. 2015, 27, 2981.

[6] a) L. Caschera, A. Lazzara, L. Piergallini, D. Ricci, B. Tuscano, A. Vanzulli, Pharmacol. Res. 2016, 110, 65; b) B. B. Goldberg, J.-B. Liu, F. Forsberg, Ultrasound Med. Biol. 1994, 20, 319; c) V. Pansare, S. Hejazi, W. Faenza, R. K. Prud'homme, Chem. Mater. 2012, 24, 812; d) S. Sinharay, M. D. Pagel, Annu. Rev. Anal. Chem. 2016, 9, 95; e) H. Lusic, M. W. Grinstaff, Chem. Rev. 2013, 113, 1641; f) J. Weber, P. C. Beard, S. E. Bohndiek, Nat. Methods 2016, 13, 639.

[7] P. P. Mathai, J. A. Liddle, S. M. Stavis, Applied Physics Review 2016, 3, 011105.

[8] Y. Zhou, B. J. Nelson, Opt. Eng. 1999, 38, 1989.

[9] H. Luo, L. Zhu, H. Ding, Sens. Actuator A-Phys. 2006, 132, 480.

[10] M. Ammi, V. Fremont, A. Ferreira, IEEE Trans. Robot. 2009, 25, 184.

[11] C. R. Copeland, J. Geist, C. D. McGray, V. A. Aksyuk, J. A. Liddle, B. R. Ilic, S. M. Stavis, Light Sci. Appl. 2018, 7, 31.

[12] L. Zhang, J. J. Abbott, L. Dong, K. E. Peyer, B. E. Kratochvil, H. Zhang, C. Bergeles, B. J. Nelson, Nano Lett. 2009, 9, 3663.

[13] M. P. Kummer, J. J. Abbott, B. E. Kratochvil, R. Borer, A. Sengul, B. J. Nelson, IEEE Trans. Robot. 2010, 26, 1006.

[14] B. E. Kratochvil, M. P. Kummer, S. Erni, R. Borer, D. R. Frutiger, S. Schürle, B. J. Nelson, in Experimental robotics: The 12th international symposium on experimental 
robotics, (Eds: O. Khatib, V. Kumar, G. Sukhatme), Springer Berlin Heidelberg, Berlin, Heidelberg 2014, 317.

[15] S. Schuerle, S. Erni, M. Flink, B. E. Kratochvil, B. J. Nelson, IEEE Trans. Magn. 2013, 49, 321.

[16] F. Ullrich, C. Bergeles, J. Pokki, O. Ergeneman, S. Erni, G. Chatzipirpiridis, S. Pane, C. Framme, B. J. Nelson, Invest. Ophthalmol. Vis. Sci. 2013, 54, 2853.

[17] a) E. Diller, S. Floyd, C. Pawashe, M. Sitti, IEEE Trans. Robot. 2012, 28, 172; b) C. Pawashe, S. Floyd, E. Diller, M. Sitti, IEEE Trans. Robot. 2012, 28, 467.

[18] W. Gao, A. Uygun, J. Wang, J. Am. Chem. Soc. 2012, 134, 897.

[19] D. R. Frutiger, K. Vollmers, B. E. Kratochvil, B. J. Nelson, International Journal of Robotics Research 2010, 29, 613.

[20] C. Bergeles, G. Fagogenis, J. J. Abbott, B. J. Nelson, "Tracking intraocular microdevices based on colorspace evaluation and statistical color/shape information", presented at IEEE Int. Conf. Robot. Autom., Kobe, Japan, 12-17 May 2009, 2009.

[21] a) J. Ens, P. Lawrence, IEEE Trans. Pattern Anal. Mach. Intell. 1993, 15, 97; b) M. Subbarao, G. Surya, Int. J. Comput. Vis. 1994, 13, 271.

[22] S. Schürle, M. S. Sakar, A. Meo, J. Möller, B. E. Kratochvil, C. S. Chen, V. Vogel, B. J. Nelson, "Three-dimensional, automated magnetic biomanipulation with subcellular resolution", presented at IEEE Int. Conf. Robot. Autom., Karlsruhe, Germany, 6-10 May 2013, 2013.

[23] a) C. Bergeles, K. Shamaei, J. J. Abbott, B. J. Nelson, "On imaging and localizing untethered intraocular devices with a stationary camera", presented at 2 nd IEEE RAS \& EMBS International Conference on Biomedical Robotics and Biomechatronics, Scottsdale, AZ, USA, 19-22 Oct. 2008, 2008; b) C. Bergeles, K. Shamaei, J. J. Abbott, B. J. Nelson, IEEE Trans. Biomed. Eng. 2010, 57, 2064.

[24] C. Bergeles, B. E. Kratochvil, B. J. Nelson, IEEE Trans. Robot. 2012, 28, 798. 
[25] T. Drummond, R. Cipolla, IEEE Trans. Pattern Anal. Mach. Intell. 2002, 24, 932.

[26] a) P. C. Lauterbur, Nature 1973, 242, 190; b) P. Vartholomeos, M. Fruchard, A. Ferreira, C. Mavroidis, Annu. Rev. Biomed. Eng. 2011, 13, 157.

[27] T. Wortmann, C. Dahmen, C. Geldmann, S. Fatikow, "Recognition and tracking of magnetic nanobots using MRI", presented at 2010 International Symposium on Optomechatronic Technologies, Toronto, ON, Canada, 25-27 Oct. 2010, 2010.

[28] O. Felfoul, E. Aboussouan, A. Chanu, S. Martel, "Real-time positioning and tracking technique for endovascular untethered microrobots propelled by MRI gradients", presented at IEEE Int. Conf. Robot. Autom., Kobe, Japan, 12-17 May 2009, 2009.

[29] a) D. Folio, C. Dahmen, T. Wortmann, M. A. Zeeshan, K. Shou, S. Pane, B. J. Nelson, A. Ferreira, S. Fatikow, "MRI magnetic signature imaging, tracking and navigation for targeted micro/nano-capsule therapeutics", presented at IEEE/RSJ International Conference on Intelligent Robots and Systems San Francisco, CA, USA, 25-30 Sept. 2011, 2011; b) C. Dahmen, D. Folio, T. Wortmann, A. Kluge, A. Ferreira, S. Fatikow, "Evaluation of a MRI based propulsion/control system aiming at targeted micro/nano-capsule therapeutics", presented at International Conference on Intelligent Robots and Systems, Vilamoura, Portugal, 7-12 Oct. 2012, 2012.

[30] a) L. Mellal, K. Belharet, D. Folio, A. Ferreira, J. Nanopart. Res. 2015, 17, 64; b) N. Olamaei, F. Cheriet, G. Beaudoin, S. Martel, "MRI visualization of a single 15 microm navigable imaging agent and future microrobot", presented at 2010 Annual International Conference of the IEEE Engineering in Medicine and Biology Society (EMBC), Buenos Aires, Argentina, 2010.

[31] X. Yan, Q. Zhou, M. Vincent, Y. Deng, J. Yu, J. Xu, T. Xu, T. Tang, L. Bian, Y.-X. J. Wang, K. Kostarelos, L. Zhang, Sci. Robotics 2017, 2, eaaq1155.

[32] K. Belharet, D. Folio, A. Ferreira, Minim. Invasive Ther. Allied Technol. 2010, 19, 157. 
[33] G. Kosa, P. Jakab, G. Szekely, N. Hata, Biomed. Microdevices 2012, 14, 165.

[34] S. Martel, M. Mohammadi, O. Felfoul, Z. Lu, P. Pouponneau, Int. J. Robot. Res. 2009, $28,571$.

[35] O. Felfoul, A. T. Becker, G. Fagogenis, P. E. Dupont, Sci. Rep. 2016, 6, 33567.

[36] a) A. Bigot, C. Tremblay, G. Soulez, S. Martel, IEEE Trans. Robot. 2014, 30, 719; b)

D. Folio, A. Ferreira, IEEE Trans. Robot. 2017, 33, 583.

[37] R. Tanaka, T. Yumoto, N. Shiba, M. Okawa, T. Yasuhara, T. Ichikawa, K. Tokunaga, I. Date, Y. Ujike, J. Neurosurg. 2012, 117, 1100.

[38] S. Martel, IEEE Control Syst. Mag. 2013, 33, 119.

[39] a) A. T. Becker, O. Felfoul, P. E. Dupont, IEEE Int. Conf. Robot. Autom. 2015, 1184;

b) S. Eslami, G. S. Fischer, S. E. Song, J. Tokuda, N. Hata, C. M. Tempany, I. Iordachita, IEEE Int. Conf. Robot. Autom. 2013, 20132, 1228; c) A. B. Rosenkrantz, S. M. Scionti, S. Mendrinos, S. S. Taneja, AJR Am. J. Roentgenol. 2011, 197, W90.

[40] a) B. Gleich, J. Weizenecker, Nature 2005, 435, 1214; b) N. Panagiotopoulos, R. L. Duschka, M. Ahlborg, G. Bringout, C. Debbeler, M. Graeser, C. Kaethner, K. Ludtke-Buzug, H. Medimagh, J. Stelzner, T. M. Buzug, J. Barkhausen, F. M. Vogt, J. Haegele, Int. J. Nanomedicine 2015, 10, 3097; c) A. Bakenecker, M. Ahlborg, C. Debbeler, C. Kaethner, K. Lüdtke-Buzug, in Precision Medicine, (Eds: H.-P. Deigner, M. Kohl), Academic Press, 2018, 183.

[41] P. W. Goodwill, A. Tamrazian, L. R. Croft, C. D. Lu, E. M. Johnson, R. Pidaparthi, R. M. Ferguson, A. P. Khandhar, K. M. Krishnan, S. M. Conolly, Appl. Phys. Lett. 2011, 98, 262502.

[42] J. Weizenecker, B. Gleich, J. Rahmer, H. Dahnke, J. Borgert, Phys. Med. Bio. 2009, $54, \mathrm{~L} 1$.

[43] a) M. H. Loser, N. Navab, in Medical Image Computing and Computer-Assisted Intervention - MICCAI 2000: Third International Conference, Pittsburgh, PA, USA, October 
11-14, 2000. Proceedings, (Eds: S. L. Delp, A. M. DiGoia, B. Jaramaz), Springer Berlin Heidelberg, Berlin, Heidelberg 2000, 887; b) N. Navab, B. Bascle, M. Loser, B. Geiger, R. Taylor, "Visual servoing for automatic and uncalibrated needle placement for percutaneous procedures", presented at IEEE Conference on Computer Vision and Pattern Recognition, 2000.

[44] M. Azizian, N. Najmaei, M. Khoshnam, R. Patel, Int. J. Med. Robot. 2015, 11, 67.

[45] K. Cha, S. Jeong, J. Choi, L. Qin, J. Li, J. Park, S. Park, "Electromagnetic actuation methods for intravascular locomotive microrobot", presented at Annual International Conference of the IEEE Engineering in Medicine and Biology, Buenos Aires, Argentina, Aug. 31 2010-Sept. 4 2010, 2010.

[46] a) S. Jeong, H. Choi, C. Lee, K. Kwon, G. Go, D. S. Sim, K. S. Lim, M. H. Jeong, S. Y. Ko, J.-o. Park, S. Park, "A study on 3D locomotion of intravascular therapeutic microrobot using EMA system", presented at International Conference on Control, Automation and Systems, Gwangju, Korea, 2013; b) S. Jeong, H. Choi, C. Lee, G. Go, D. S. Sim, K. S. Lim, M. H. Jeong, S. Y. Ko, J.-o. Park, S. Park, Int. J. Control Autom. Syst. 2015, 13, 1465.

[47] K. Jae-Hee, K. Kyu-Gyeom, L. Hee-Sin, P. Dong-Jun, K. Kyong-Woo, "Development of bi-plane X-ray imaging system for real-time position recognition of intravascular therapeutic microrobot using EMA system", presented at 44th International Symposium on Robotics, Seoul, South Korea, 24-26 Oct. 2013, 2013.

[48] A. Hong, A. J. Petruska, B. J. Nelson, "Tracking a magnetically guided catheter with a single rotating C-Arm", presented at IEEE Int. Conf. Robot. Autom., Seattle, WA, USA, 26-30 May 2015, 2015.

[49] T. Beyer, D. W. Townsend, T. Brun, P. E. Kinahan, M. Charron, R. Roddy, J. Jerin, J. Young, L. Byars, R. Nutt, J. Nucl. Med. 2000, 41, 1369.

[50] T. F. Lang, B. H. Hasegawa, S. C. Liew, J. K. Brown, S. C. Blankespoor, S. M. Reilly, E. L. Gingold, C. E. Cann, J. Nucl. Med. 1992, 33, 1881. 
[51] D. Vilela, U. Cossio, J. Parmar, A. M. Martinez-Villacorta, V. Gomez-Vallejo, J. Llop, S. Sanchez, ACS Nano 2018, 12, 1220.

[52] D. C. Ackland, F. Keynejad, M. G. Pandy, Proc. Inst. Mech. Eng. H 2011, 225, 1136.

[53] a) S. Kunjachan, J. Ehling, G. Storm, F. Kiessling, T. Lammers, Chem. Rev. 2015, 115, 10907; b) S. N. Narouze, Atlas of Ultrasound-Guided Procedures in Interventional Pain Management, Springer-Verlag New York, 2018.

[54] M. Fluckiger, A. Neild, B. J. Nelson, Ultrasonics 2012, 52, 447.

[55] I. S. M. Khalil, P. Ferreira, R. Eleuterio, C. L. de Korte, S. Misra, "Magnetic-based closed-loop control of paramagnetic microparticles using ultrasound feedback", presented at 2014 IEEE International Conference on Robotics and Automation, Hong Kong, China, 2014.

[56] I. S. Khalil, V. Magdanz, S. Sanchez, O. G. Schmidt, L. Abelmann, S. Misra, Conference proceedings: Annual International Conference of the IEEE Engineering in Medicine and Biology Society 2013, 2013, 5299.

[57] A. Sanchez, V. Magdanz, O. G. Schmidt, S. Misra, "Magnetic control of self-propelled microjets under ultrasound image guidance", presented at 5th IEEE RAS \& EMBS International Conference on Biomedical Robotics and Biomechatronics, São Paulo, Brazil, 12-15 Aug. 2014, 2014.

[58] I. S. M. Khalil, H. C. Dijkslag, L. Abelmann, S. Misra, Appl. Phys. Lett. 2014, 104.

[59] V. Chan, A. Perlas, in Atlas of ultrasound-guided procedures in interventional pain management, (Ed: S. N. Narouze), 2011.

[60] S. Scheggi, K. K. T. Chandrasekar, C. Yoon, B. Sawaryn, G. van de Steeg, D. H. Gracias, S. Misra, "Magnetic motion control and planning of untethered soft grippers using ultrasound image feedback", presented at 2017 IEEE International Conference on Robotics and Automation, Singapore, 2017.

[61] a) L. Rubinstein, "A practical nanorobot for treatment of various medical problems", presented at Eighth Foresight Conference on Molecular Nanotechnology, Maryland, Nov. 3-5 
2000, 2000; b) M. Flueckiger, B. J. Nelson, "Ultrasound emitter localization in heterogeneous media", presented at Annual International Conference of the IEEE Engineering in Medicine and Biology Society, Lyon, France, 22-26 Aug. 2007, 2007; c) Z. Nagy, M. Fluckiger, O. Ergeneman, S. Pane, M. Probst, B. J. Nelson, "A wireless acoustic emitter for passive localization in liquids", presented at 2009 IEEE International Conference on Robotics and Automation, Kobe, Japan, 2009.

[62] a) M. Flückiger, Z. Nagy, M. Probst, O. Ergeneman, S. Pané, B. J. Nelson, Sens. Actuator A-Phys. 2009, 154, 109; b) M. Probst, M. Fluckiger, S. Pane, O. Ergeneman, Z. Nagy, B. J. Nelson, IEEE Trans. Ind. Electron. 2009, 56, 2657.

[63] I. Steinberg, M. Ben-David, I. Gannot, Nanomed. Nanotech. Biol. Med. 2012, 8, 569.

[64] A. Tsalach, I. Steinberg, I. Gannot, IEEE Trans. Biomed. Eng. 2014, 61, 2313.

[65] V. Garcia-Gradilla, J. Orozco, S. Sattayasamitsathit, F. Soto, F. Kuralay, A. Pourazary, A. Katzenberg, W. Gao, Y. Shen, J. Wang, ACS Nano 2013, 7, 9232.

[66] T. Xu, F. Soto, W. Gao, V. Garcia-Gradilla, J. Li, X. Zhang, J. Wang, J. Am. Chem. Soc. 2014, 136, 8552.

[67] D. Ahmed, C. Dillinger, A. Hong, B. J. Nelson, Adv. Mater. Technol. 2017, 2, 1700050.

[68] A. L. Balk, L. O. Mair, P. P. Mathai, P. N. Patrone, W. Wang, S. Ahmed, T. E. Mallouk, J. A. Liddle, S. M. Stavis, ACS Nano 2014, 8, 8300.

[69] M. Rafat, F. F. Li, P. Fagerholm, N. S. Lagali, M. A. Watsky, R. Munger, T. Matsuura, M. Griffith, Biomaterials 2008, 29, 3960.

[70] R. Naccache, E. M. Rodriguez, N. Bogdan, F. Sanz-Rodriguez, C. Cruz Mdel, A. J. Fuente, F. Vetrone, D. Jaque, J. G. Sole, J. A. Capobianco, Cancers 2012, 4, 1067.

[71] a) D. J. Hardee, L. Kovalchuke, T. H. Lambert, J. Am. Chem. Soc. 2010, 132, 5002; b) X. L. Lu, S. R. V. Castrillon, D. L. Shaffer, J. Ma, M. Elimelech, Environ. Sci. Technol. 2013, $47,12219$. 
[72] J. R. McCarthy, R. Weissleder, Adv. Drug Deliv. Rev. 2008, 60, 1241.

[73] a) M. Garcia, J. Orozco, M. Guix, W. Gao, S. Sattayasamitsathit, A. Escarpa, A. Merkoci, J. Wang, Nanoscale 2013, 5, 1325; b) B. Jurado-Sanchez, A. Escarpa, J. Wang, Chem. Commun. 2015, 51, 14088.

[74] J. C. Love, L. A. Estroff, J. K. Kriebel, R. G. Nuzzo, G. M. Whitesides, Chem. Rev. $\mathbf{2 0 0 5}, 105,1103$.

[75] C.-J. Pan, Y.-X. Dong, K. D. Jandt, J. Biomater. Nanobiotechnol. 2012, 03, 353.

[76] D. Tarn, C. E. Ashley, M. Xue, E. C. Carnes, J. I. Zink, C. J. Brinker, Acc. Chem. Res. 2013, 46, 792 .

[77] E. Saxon, J. I. Armstrong, C. R. Bertozzi, Org. Lett. 2000, 2, 2141.

[78] a) F. Liu, Y. Liang, K. N. Houk, Acc. Chem. Res. 2017, 50, 2297; b) N. K. Devaraj, ACS Cent. Sci. 2018, 4, 952; c) C. S. McKay, M. G. Finn, Chem. Biol. 2014, 21, 1075.

[79] A. M. Maier, C. Weig, P. Oswald, E. Frey, P. Fischer, T. Liedl, Nano Lett. 2016, 16, 906.

[80] M. Hoop, F. Mushtaq, C. Hurter, X.-Z. Chen, B. J. Nelson, S. Pané, Nanoscale 2016, $8,12723$.

[81] B. E. F. de Avila, A. Martin, F. Soto, M. A. Lopez-Ramirez, S. Campuzano, G. M. Vasquez-Machado, W. W. Gao, L. F. Zhang, J. Wang, ACS Nano 2015, 9, 6756.

[82] B. Jurado-Sanchez, A. Escarpa, Trends Anal. Chem. 2016, 84, 48.

[83] B. Wang, Y. Zhang, L. Zhang, Quant. Imaging Med. Surg. 2018, 8, 461.

[84] M. E. Gindy, R. K. Prud'homme, Expert Opin. Drug Deliv. 2009, 6, 865.

[85] J. V. Frangioni, Curr. Opin. Chem. Biol. 2003, 7, 626.

[86] a) G. M. van Dam, G. Themelis, L. M. A. Crane, N. J. Harlaar, R. G. Pleijhuis, W. Kelder, A. Sarantopoulos, J. S. de Jong, H. J. G. Arts, A. G. J. van der Zee, J. Bart, P. S. Low, V. Ntziachristos, Nat. Med. 2011, 17, 1315; b) Z. J. Deng, S. W. Morton, E. Ben-Akiva, E. C. Dreaden, K. E. Shopsowitz, P. T. Hammond, ACS Nano 2013, 7, 9571. 
[87] A. Servant, F. M. Qiu, M. Mazza, K. Kostarelos, B. J. Nelson, Adv. Mater. 2015, 27, 2981.

[88] X. H. Zheng, D. Xing, F. F. Zhou, B. Y. Wu, W. R. Chen, Mol. Pharm. 2011, 8, 447.

[89] K. Li, B. Liu, Chem. Soc. Rev. 2014, 43, 6570.

[90] W. Gao, J. Wang, ACS Nano 2014, 8, 3170.

[91] M. Medina-Sanchez, O. G. Schmidt, Nature 2017, 545, 406.

[92] P. Cai, B. Hu, W. R. Leow, X. Wang, X. J. Loh, Y. L. Wu, X. Chen, Adv. Mater. 2018, 30, e1800572.

[93] a) B. J. Nelson, K. E. Peyer, ACS Nano 2014, 8, 8718; b) D. Schamel, A. G. Mark, J. G. Gibbs, C. Miksch, K. I. Morozov, A. M. Leshansky, P. Fischer, ACS Nano 2014, 8, 8794. 

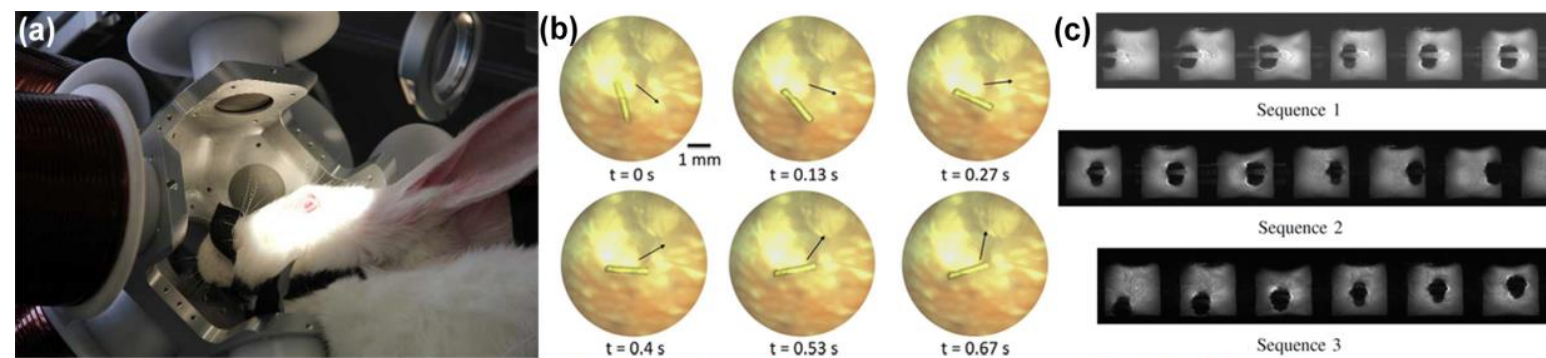

Sequence 1

(d)
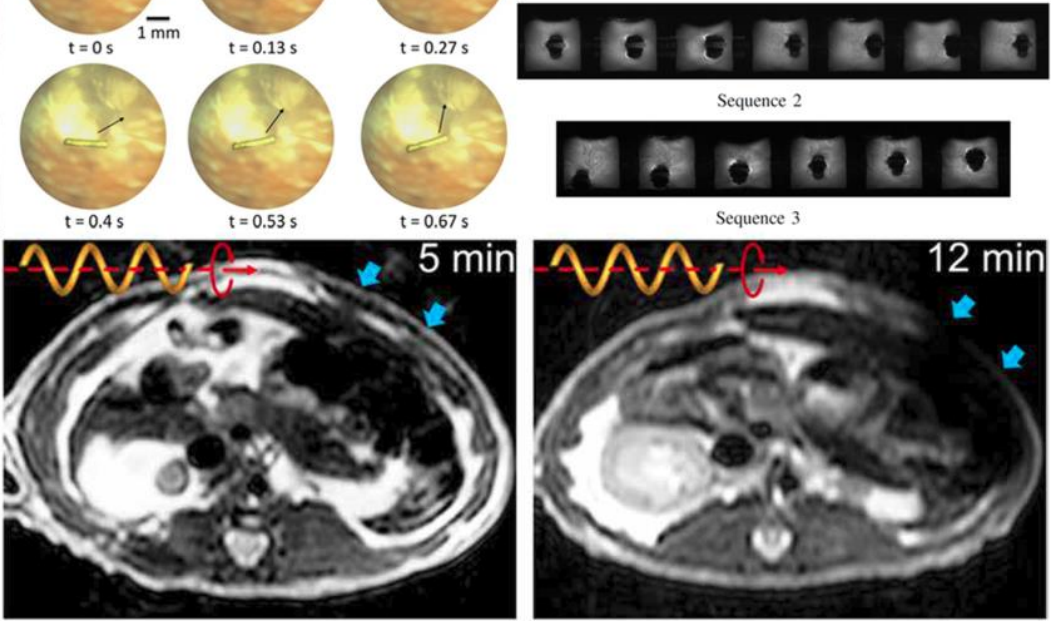

Sequence 2
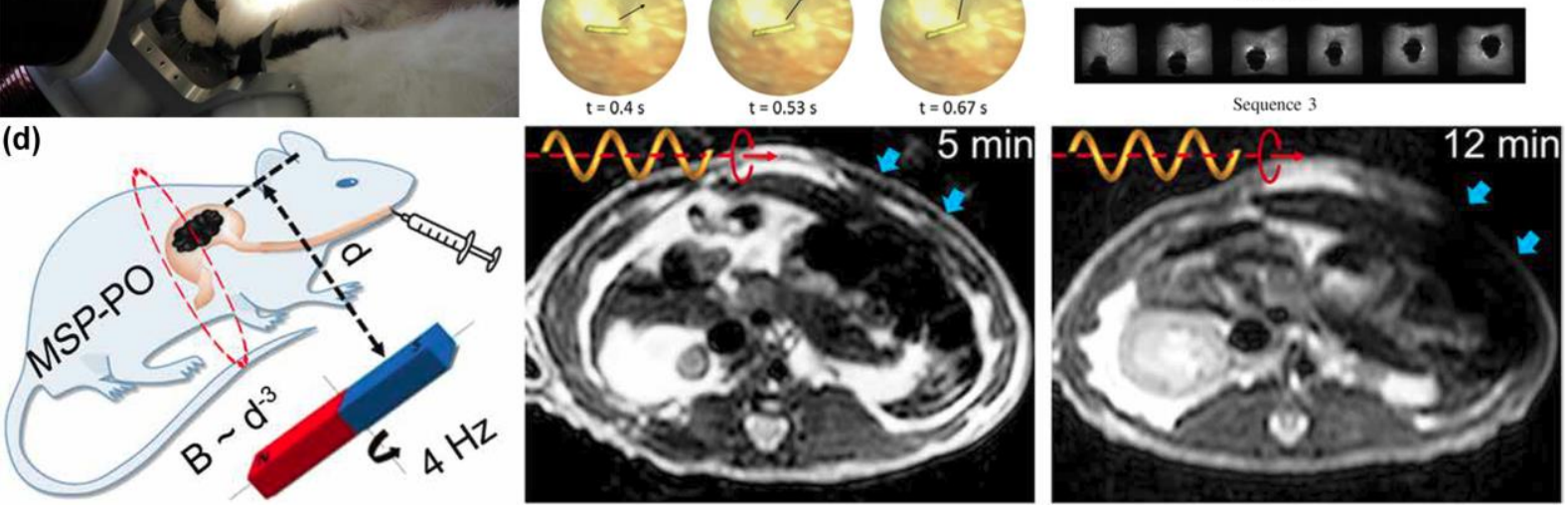

Figure 1. (a) A rabbit with the eye of interest placed centrally in the OctoMag workspace. (b) A microrobot (gold) inside the vitreous of a live rabbit is rotated by an applied magnetic field (black arrow). Adapted from ${ }^{[16]}$ with permission. (c) MRI-based navigation and tracking of a

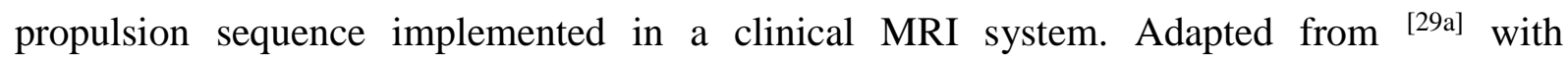
permission. (d) T2-weighted cross-sectional MR imaging of magnetized S. platensis (MSP) swarms inside rats. MSP swarm subject to actuation and steering (with a rotating magnetic field) of different time periods (5 min and $12 \mathrm{~min}$, respectively) before MR imaging across the rat's stomach. Adapted from ${ }^{[31]}$ with permission. Copyright AAAS. 


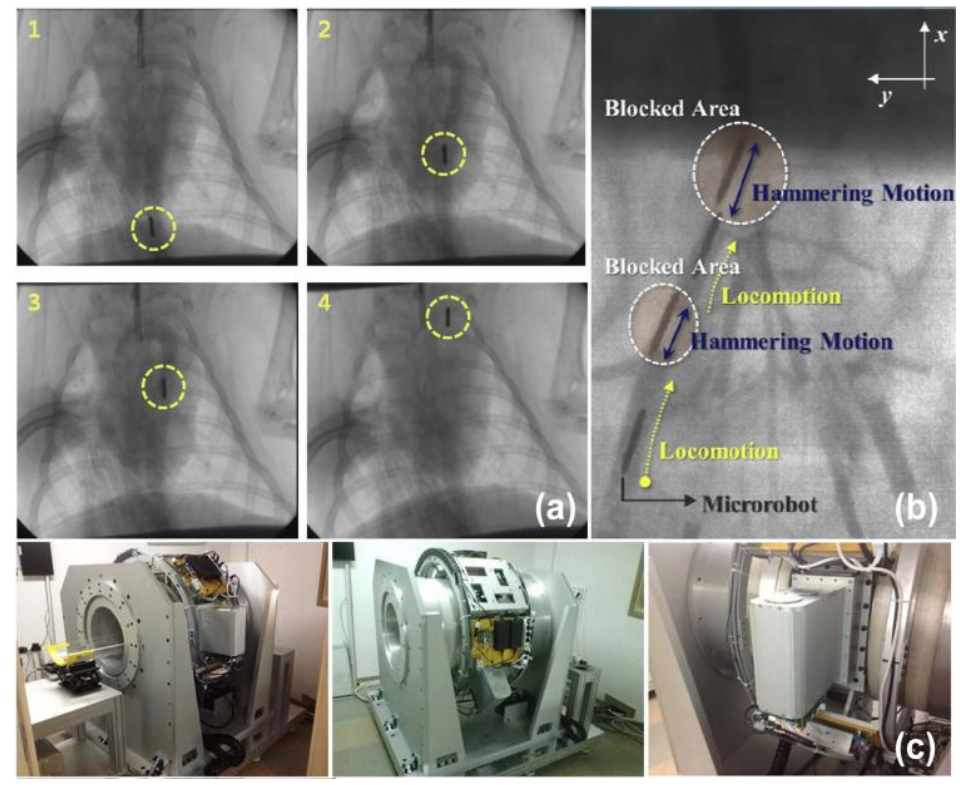

Figure 2. (a) The microrobot going upstream against the blood pressure in the rabbit aorta. The yellow dotted line marks the micro robot. Reproduced from ${ }^{[45]}$. Copyright IEEE. (b) In vivo experiment of microrobot in blood vessel of live mini-pig: locomotion and tunneling performances. Reproduced from ${ }^{[46 b]}$. Copyright Springer. (c) Bi-plane X-ray imaging system integrated in EMA system. Reproduced from ${ }^{[47]}$. Copyright IEEE. 

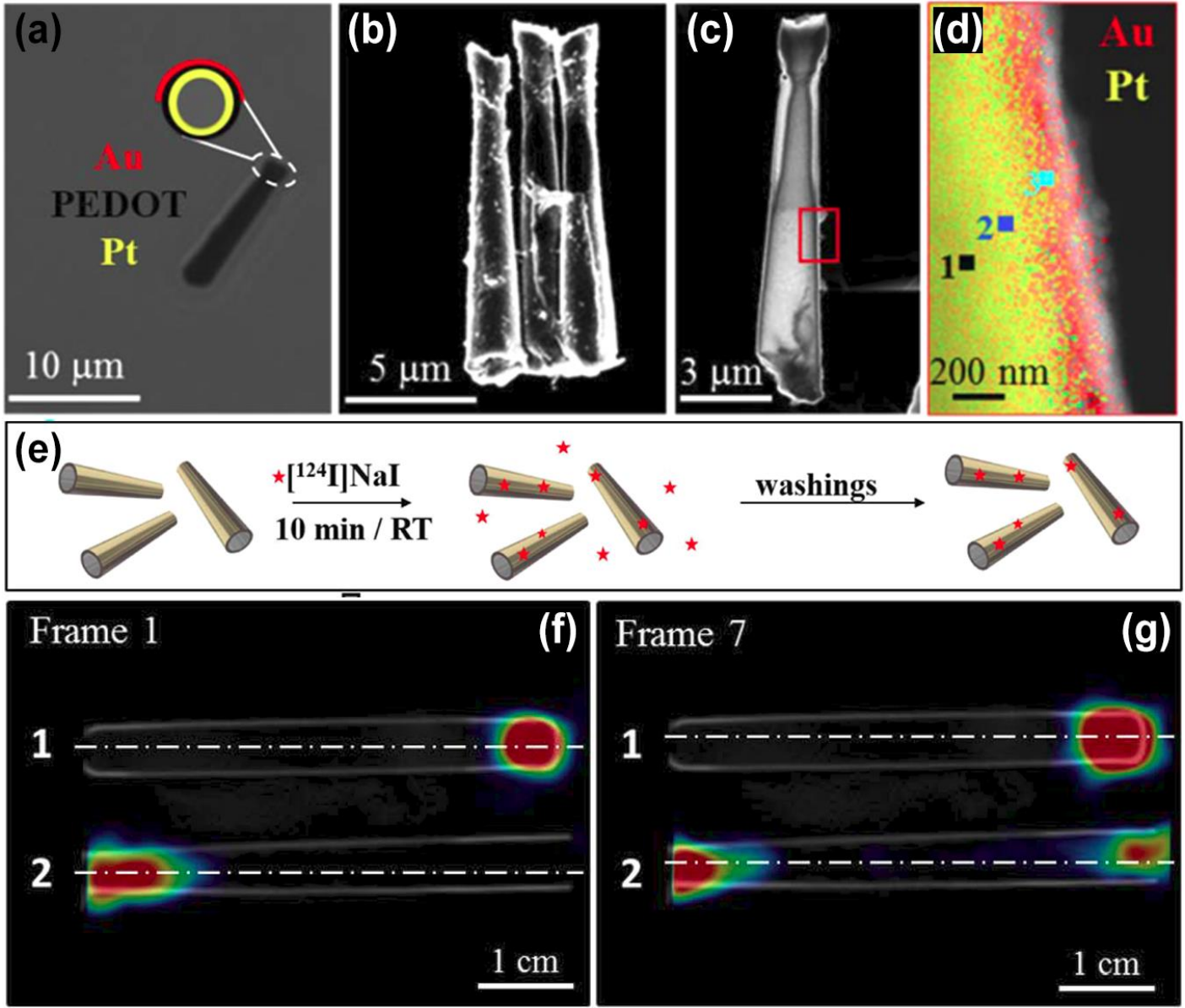

Figure 3. Characterization of the Au-micromotors through (a) optical imaging, (b) SEM imaging, (c) STEM imaging and (d) TEMEDXS mapping of Au (red) and Pt (yellow) using a TEM copper grid (enlarging part of the micromotor surface framed by the red rectangle in c). (e) Schematic illustration of labeling of Au-micromotors with ${ }^{124}$ I. (f) PET-CT imaging of Au-micromotors corresponding to frame 1 (acquired in the time range $0 \mathrm{~min}<\mathrm{t}<1 \mathrm{~min}$ ) and frame 7 (10 $\min <\mathrm{t}<15 \mathrm{~min})$. Phantom 1 was filled with SDS solution; phantom 2 with SDS/ $\mathrm{H}_{2} \mathrm{O}_{2}$. Trajectories followed to obtain radioactivity profiles are shown as a white dotted line. Adapted from ${ }^{[51]}$. Copyright American Chemical Society. 

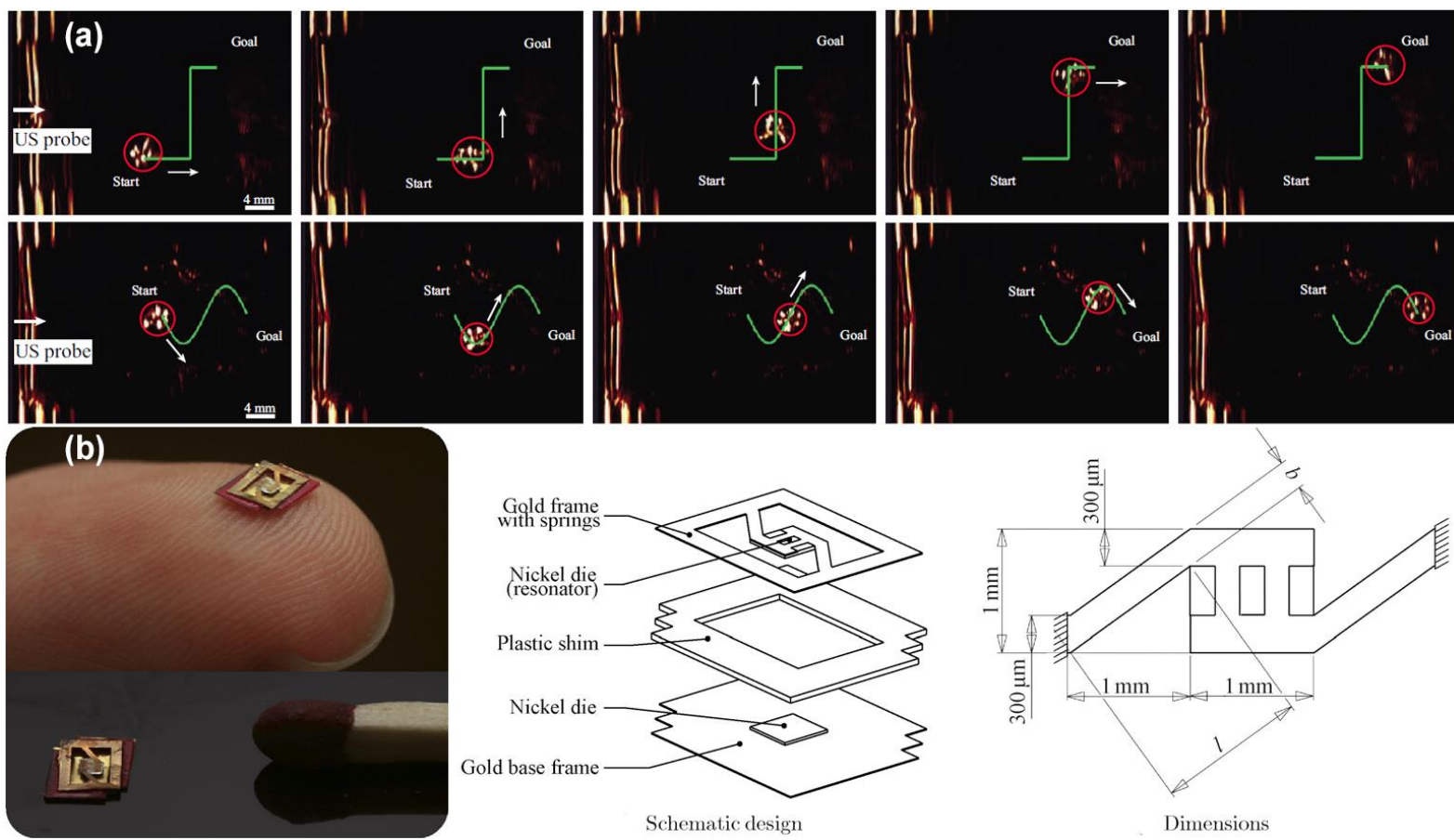

Figure 4. (a) Magnetic closed-loop motion control of a soft miniaturized gripper using US feedback. Reproduced from ${ }^{\left[{ }^{[0]}\right.}$. Copyright IEEE. (b) Digital image of the micro-fabricated wireless acoustic emitter and its schematic design. Adapted from ${ }^{[62 a]}$. Copyright Elsevier. 
(a)

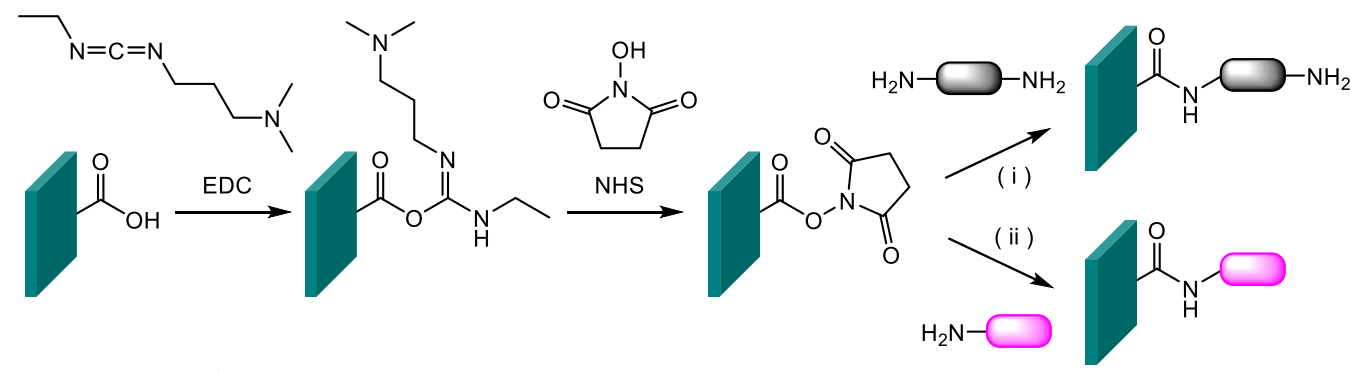

(b)

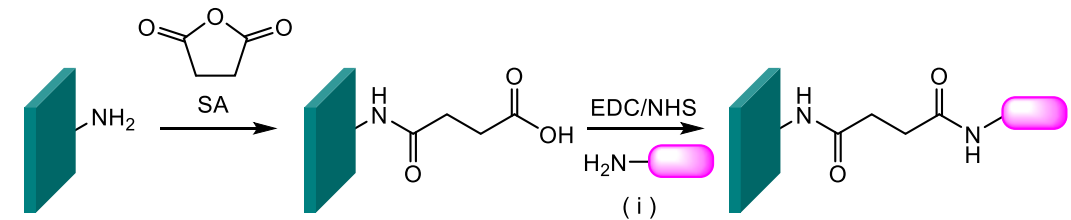

(c)

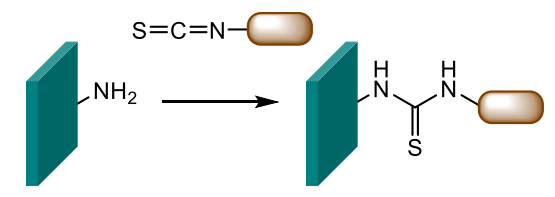

(d)

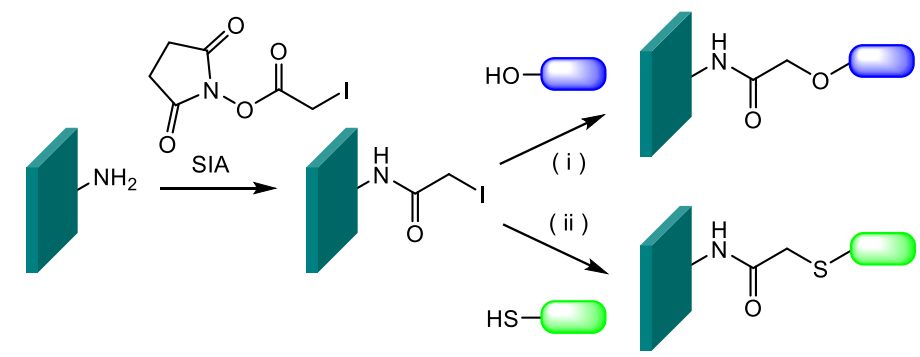

(e)

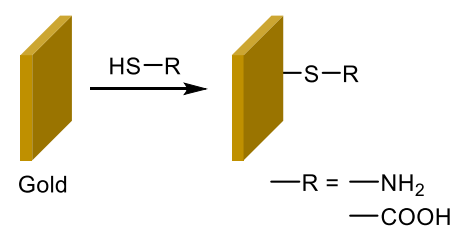

(f)

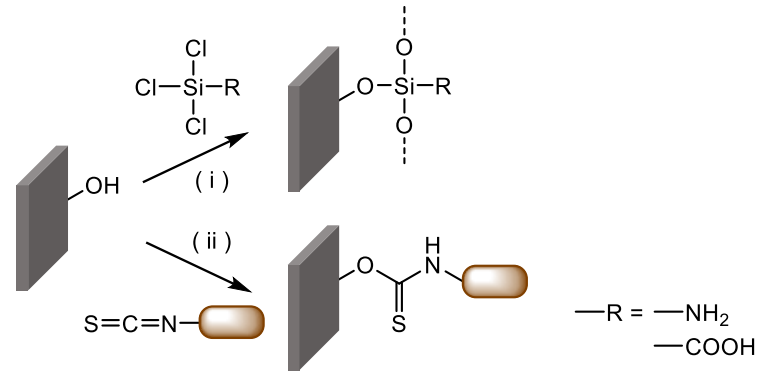

Figure 5. Synthetic routes used to derivatize and functionalize carboxylic acid (a) and amine coated surfaces (b). Derivatization of amine coated surfaces with ligands (or surfaces) bearing isothiocyanate (c) or hydroxyl and sulfhydryl groups (d). Schematic illustration showing the strategies used for the functionalization of gold (e) and titanium (f) surfaces. 
(a)

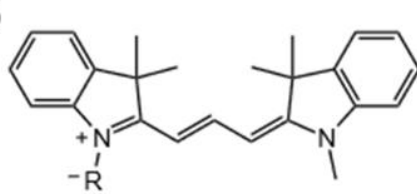

(b) $\circ$

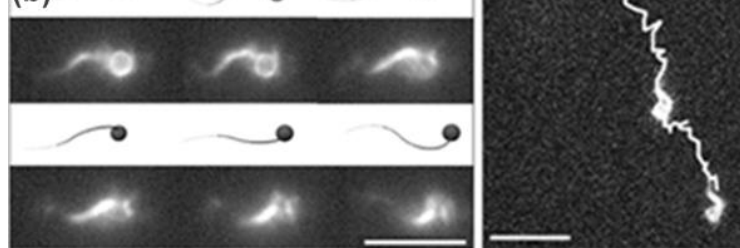

(d)

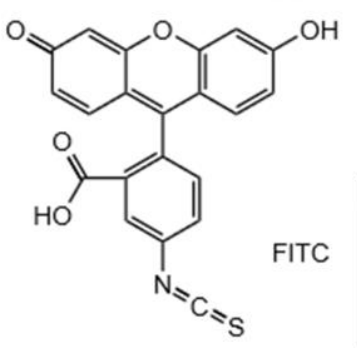

(e)
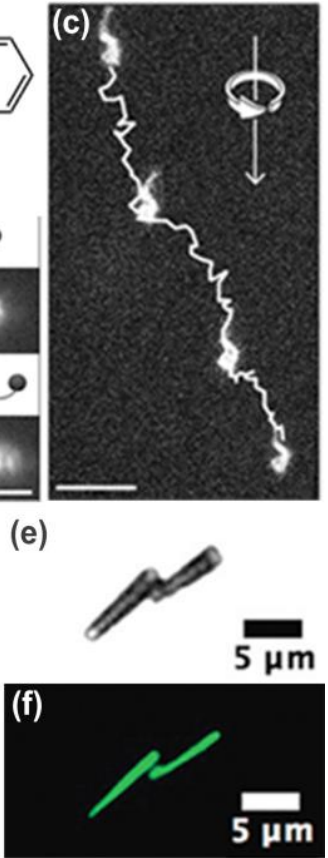

Figure 6. (a) Chemical structure of $\mathrm{Cy} 3$, and in (b) fluorescence microscopy images of the magnetically propelled DNA-based microstructures. In (c) superimposed fluorescence micrographs showing the motion of these hybrid structures. Adapted from ${ }^{[79]}$. Copyright American Chemical Society. (d) Chemical structure of FITC. (e) and (f) Optical and fluorescence microscopy images of functionalized nanotubes with a FITC tagged ssDNA. Adapted from ${ }^{[80]}$. Copyright Royal Chemical Society. 
(a)
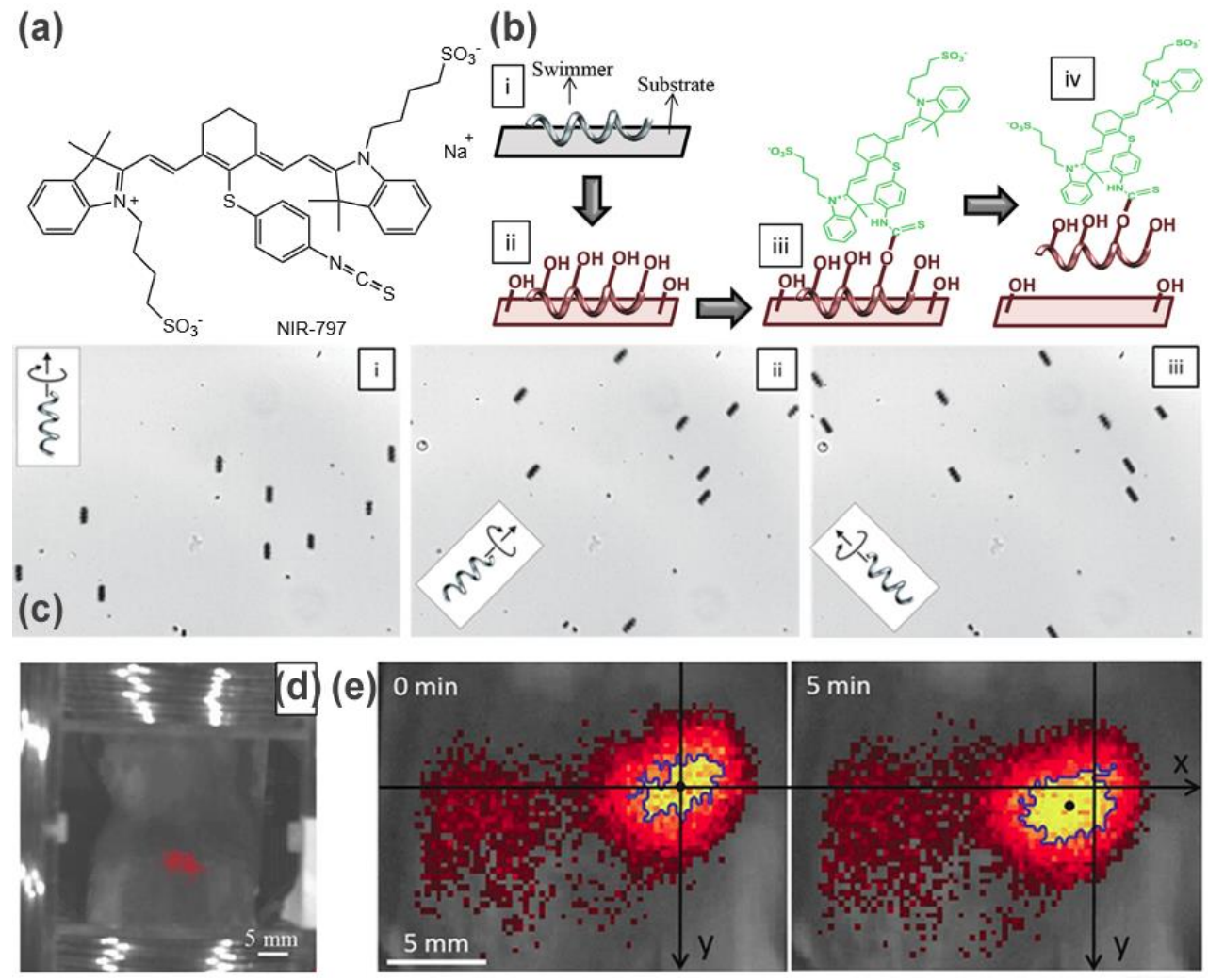

Figure 7. (a) Chemical structure of NIR-797. (b) Schematic drawing of the functionalization of ABFs with NIR-797. (c) Optical micrographs showing the controlled movement of ABFs in vitro and under rotating magnetic fields. (d) The infrared fluorescent image of the ABFs in a mouse. (e) Infrared fluorescent images showing a swarm of ABFs swimming under the actuation of a rotating magnetic field. Adapted from ${ }^{[87]}$ with permission. Copyright Wiley. 


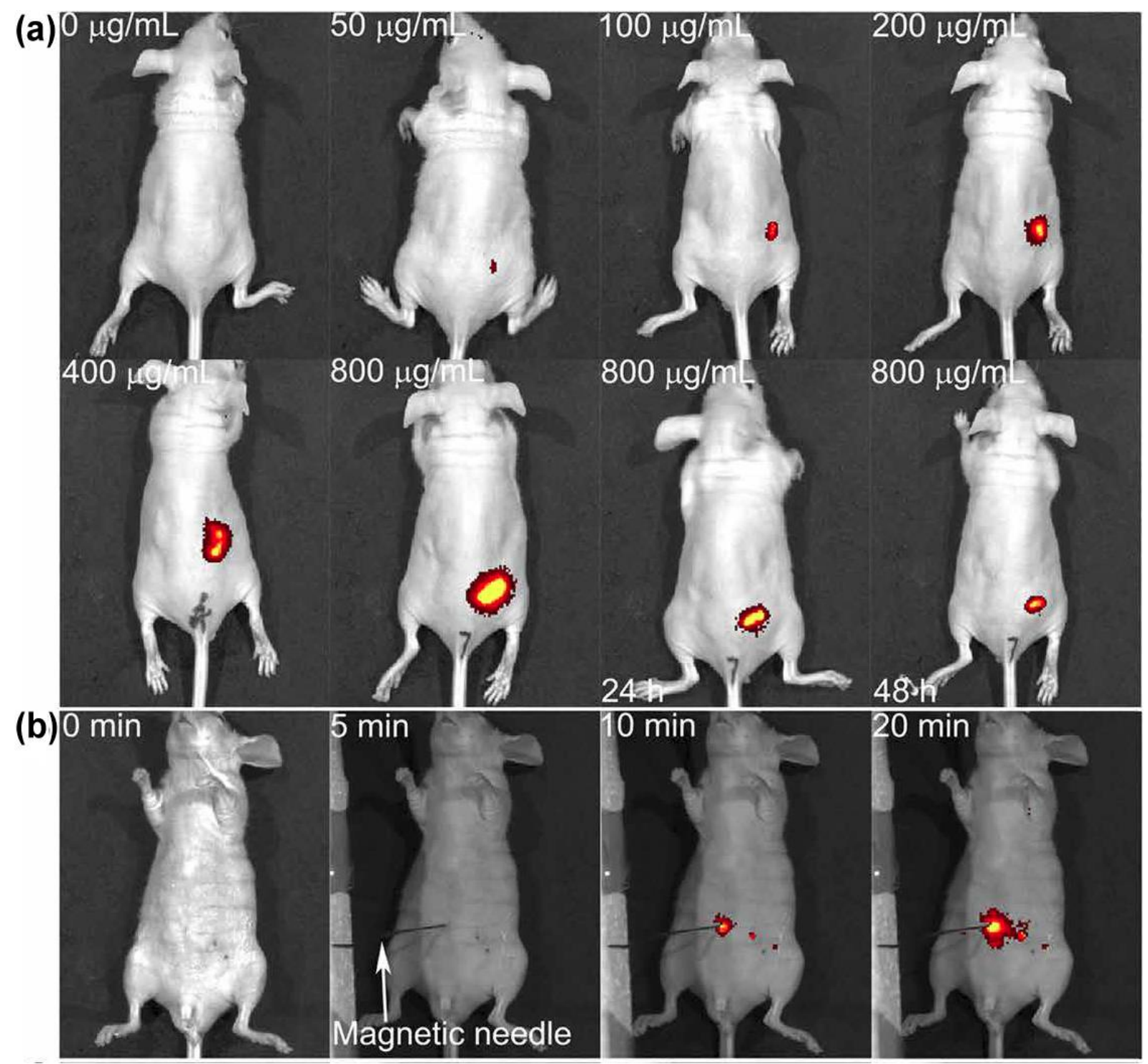

Figure 8. Fluorescence-based in vivo imaging of MSP. (a) Fluorescence of $100 \mu$ of MSP with varied concentrations in the subcutaneous tissue of nude Balb/c athymic mice at three residence times. The residence time is 0 min unless otherwise specified. (b) Fluorescence of $300 \mu 1$ of MSP in the intraperitoneal cavity at various residence times. Adapted from ${ }^{[31]}$ with permission. Copyright AAAS. 
Table 1. Comparison of imaging modalities for tracking micro- and nanorobots

\begin{tabular}{|c|c|c|}
\hline & Advantages & Limitations \\
\hline $\begin{array}{l}\text { Optical } \\
\text { Tracking }\end{array}$ & $\begin{array}{l}\text { Real time observation; } \\
\text { Simple equipment, low cost; } \\
\text { High spatial resolution }\end{array}$ & $\begin{array}{l}\text { 3-D localization of microrobots is hard; } \\
\text { Easily interfered by noise or workspace } \\
\text { contamination; } \\
\text { Can only be used in transparent } \\
\text { microenvironment such as vitreous humor in } \\
\text { eyes }\end{array}$ \\
\hline MRI & $\begin{array}{l}\text { Magnetic field is biocompatible non- } \\
\text { ionizing radiation; } \\
\text { Excellent tissue contrast; } \\
\text { 3-D localization ability; } \\
\text { Not only used for imaging but also } \\
\text { used to manipulate microrobots }\end{array}$ & $\begin{array}{l}\text { Magnetic materials will cause artifacts; } \\
\text { Real time acquisition is challenging; } \\
\text { High cost } \\
\text { Low spatial resolution; }\end{array}$ \\
\hline MPI & $\begin{array}{l}\text { Magnetic field is biocompatible non- } \\
\text { ionizing radiation; } \\
\text { 3-D localization ability; } \\
\text { High spatial resolution; } \\
\text { Fast scanning speed }\end{array}$ & $\begin{array}{l}\text { Limited to superparamagnetic nanoparticles; } \\
\text { Only small machines for research and pre- } \\
\text { clinical applications; }\end{array}$ \\
\hline $\begin{array}{l}\text { X-ray } \\
\text { Fluoroscopy }\end{array}$ & $\begin{array}{l}\text { Fluoroscopy is real-time imaging; } \\
\text { High sensitivity; Fluoroscopy can } \\
\text { distinguish materials with density } \\
\text { difference less than } 1 \% \text {; } \\
\text { Large penetration depth of human } \\
\text { body; }\end{array}$ & $\begin{array}{l}\text { Low spatial resolution; } \\
\text { Ionizing radiation }\end{array}$ \\
\hline $\begin{array}{l}\text { X-ray } \\
\text { Computed } \\
\text { Tomography }\end{array}$ & $\begin{array}{l}\text { Large penetration depth of human } \\
\text { body; }\end{array}$ & $\begin{array}{l}\text { Low spatial resolution; } \\
\text { Ionizing radiation; } \\
\text { Slow scanning speed }\end{array}$ \\
\hline Ultrasound & $\begin{array}{l}\text { Real time imaging; } \\
\text { Low cost; } \\
\text { Minimum adverse health effects; } \\
\text { Not only used for imaging but also } \\
\text { used for the manipulation of micro- } \\
\text { and nanorobots }\end{array}$ & $\begin{array}{l}\text { Low signal-to-noise ratio; } \\
\text { Strong scattering from bones and air pockets; } \\
\text { Limited scanning area of the patient; } \\
\text { Limited penetration depth in human body ( } \\
\text { a few cm); } \\
\text { Low special resolution in commercial } \\
\text { instruments; }\end{array}$ \\
\hline Fluorescence & $\begin{array}{l}\text { Planar resolution is high; } \\
\text { Sensitivity is high; } \\
\text { Real time imaging; }\end{array}$ & $\begin{array}{l}\text { 3-D localization of microrobots is hard; } \\
\text { Image depth is shallow; } \\
\text { Usually requires extra surface modification } \\
\text { of microstructures; } \\
\text { Limited penetration depth in human body ( } \\
\text { a few } \mathrm{cm} \text { ); }\end{array}$ \\
\hline
\end{tabular}

